\title{
Analysis of three-dimensional curved beams using isogeometric approach
}

\author{
Guodong Zhang ${ }^{1}$, Ryan Alberdi ${ }^{1}$ and Kapil Khandelwal ${ }^{2}$
}

\begin{abstract}
There is an increasing trend among architects to utilize curved structural members as opposed to traditional straight structural members in the design of modern space structures. To handle the complex geometrical forms, new structural analysis methods are needed to accurately and efficiently analyze these structures. To address this issue, an isogeometric approach is presented in this paper for the formulation of finite elements for arbitrary spatially curved 3-D beams. These elements employ the same Non-Rational B-Spline (NURBS) interpolations as used in the CAD representations to interpolate both geometry and unknown fields in the finite element analysis, leading to a seamless transition between complex CAD models utilized by architects and the analysis models used by engineers. The effectiveness of the proposed method is demonstrated on a variety of curved structural geometries of varying complexities.
\end{abstract}

Keywords: Curved beams; Isogeometric analysis (IGA); Finite element analysis (FEA); Computer aided design (CAD); Non-Rational B-Spline (NURBS).

\footnotetext{
${ }^{1}$ Graduate Students, Dept. of Civil \& Env. Engg. \& Earth Sci., University of Notre Dame

${ }^{2}$ Associate Professor, Dept. of Civil \& Env. Engg. \& Earth Sci., 156 Fitzpatrick Hall, University of Notre Dame, Notre Dame, IN 46556, United States
} 


\section{Introduction}

Design of novel structural systems requires close cooperation between architects and engineers. The architects are primarily responsible for discovering structural forms while structural engineers are responsible for the analysis and design of those forms. In this iterative design process, computer aided design (CAD) software is used by architects to discover structural forms. Structural engineers, on the other hand, use finite element methods for analysis and design of those structural forms. This process requires $\mathrm{CAD}$ designs generated by the architects to be converted into analysis suitable models that can be used in the structural finite element analysis (FEA). This is a nontrivial process especially for the design of modern structural systems. There is an increasing trend among architects to turn away from traditional structural forms, utilizing straight beam-column members, and towards increasingly complex spatial designs, utilizing curved structural members. Moreover, when design changes are made both the CAD and the FEA models have to be updated. This is a difficult task, as geometries in CAD and FEA models are represented in fundamentally different ways. In CAD, geometries are usually represented by Non-Uniform Rational B-Splines (NURBS), while in traditional FEA geometries they are approximated by linear or quadratic Lagrangian interpolations $[1,2]$. Moreover, complex geometrical forms of spatially curved beam members involving non-uniform curvature and torsion cannot be exactly represented in traditional FEA using linear or quadratic interpolations. Furthermore, straight beam theories are used when approximations are made with linear elements, which may lead to additional FEA error. To address these issues, new analysis methods are needed that can: (a) exactly represent the CAD geometries of curved 3-D structural forms, and (b) accurately describe the mechanical response of curved structural members. 
Isogeometric analysis (IGA) is introduced by Hughes et al. [3] as a way of bridging the gap between $\mathrm{CAD}$ and computer aided engineering (CAE) such as finite element analysis. IGA employs Non-uniform Rational B-Splines (NURBS) as the basis for interpolating both geometry and solution spaces. As NURBS are developed to represent curves and surfaces in CAD, IGA uses the same geometrical representation as a model derived from CAD, thus allowing structural analysis to be performed directly on the exact CAD geometry. An additional advantage of using IGA is that the order and continuity of interpolation basis can be increased efficiently and robustly through $k$-refinement $[3,4]$. This is a valuable property for interpolation of fields that require highorder continuity, and leads to results with higher accuracy [5, 6]. Moreover, even if a solution space requires only $C^{0}$-continuity, a higher degree of continuity of interpolation functions may still lead to more accurate results because of the increased smoothness of solution spaces that provide significantly better numerical approximation. In the past, IGA has been successfully used in the analysis of static problems $[3,4]$, wave propagation and fluid-structure interaction problems [4], structural optimization [7], free and forced vibration of linear and nonlinear structures $[8,9]$ and so on. In this study, the use of isogeometric analysis is explored for curved 3-D beam structures.

The mechanics of 3-D curved beams has been studied for many decades and is well understood. Most common beam formulations are based on the differential geometry of curves in $\mathbb{R}^{2}$ or $\mathbb{R}^{3}$ and are developed in the so-called "natural" coordinate system. In general, the closed form stiffness matrices are not available for arbitrary curved beam elements; however, attempts have been made to derive closed form stiffness matrices for simple circular and parabolic 2-D geometries [10, 11]. In some recent studies, direct numerical integration of the 3-D beams in the global system have been proposed, however, arbitrary 3-D spatially curved beams cannot be handled by this method 
[12]. In most cases, numerical methods based finite element approximations are used to describe the behavior of arbitrary curved beams. The standard displacement based Lagrangian FEA formulations suffer from shear and membrane locking issues, however. To address these locking issues, mixed and hybrid finite elements for 2-D and 3-D curved beams have been proposed [1316]. Nevertheless, the CAD geometries are still approximated in these methods. Some recent studies have investigated locking issues in IGA based 2-D curved beams with simple geometries $[17,18]$. As these studies were mostly focused on locking issues, only simple geometries for 2-D cases were employed and relevant implementation details for 3-D spatial curve beams are missing. Thus, to the best knowledge of the authors the use of Galerkin based IGA for arbitrary spatially curved 3-D beams has not been explored.

In this study, the isogeometric approach is used to formulate finite elements utilizing the theory of curved beams in space. Using IGA, the structural analysis is performed directly on the exact geometrical description of curved members given by the CAD models. Thus, an arbitrarily curved beam member that can be represented in CAD can be effectively analyzed using the proposed isogeometric approach. In addition to reducing modeling errors that come from geometrical and mechanical approximations, this eliminates the amount of time needed to be spent for updating the finite element models after geometrical changes are made in CAD designs. The locking issues in the proposed formulation are handled by using high order NURBS interpolations, and the effectiveness of the proposed method is demonstrated on a variety of curved beam geometries of varying complexities including geometries with non-uniform torsion and curvature. All the relevant implementation details for a general 3-D case that are needed for practical engineering applications are provided, and a potential implementation pitfall due to the discontinuity of the Frenet-Serret basis is pointed out and is appropriately addressed. The paper is organized as follows: 
Section 2 discusses the theory of curved beams in space. Section 3 gives background on IGA, while Section 4 presents the element formulation and implementation details. A locking case study and different examples of curved beams are presented in Section 5. Finally, the important conclusions of this paper are discussed in Section 6.

\section{Curved Beam Theory in 3-D}

\subsection{Frenet-Serret Frame and Geometry of Curved Beams}

Consider an arbitrary regular curve $\boldsymbol{r}(s):[0, L] \rightarrow \mathbb{R}^{3}$ embedded in $\mathbb{R}^{3}$ and parameterized by arclength parameter $s$. For a 3-D curved beam, this curve represents the line of centroids of the cross sections of the beam member. Associated with the curve is the unit orthonormal vector triad, $\{\boldsymbol{t}, \boldsymbol{n}, \boldsymbol{b}\}$, called the Frenet-Serret frame field (Eq. (1)). Note that $\boldsymbol{t}$ is the tangent vector to the curve, while $\boldsymbol{n}$ is the normal vector and $\boldsymbol{b}$ is the binormal vector. This vector field forms a local orthonormal basis, and geometrical or physical quantities of interest can be conveniently expressed in this basis. Two intrinsic properties: curvature $(\kappa(s))$, the measure of the failure of a curve to be a straight line and torsion $(\tau(s))$, the measure of the failure of a curve to lie in a plane are given by Eq. (2). Finally, the derivatives of Frenet-Serret basis, i.e. $\frac{d t}{d s}, \frac{d n}{d s}$ and $\frac{d b}{d s}$ can be expressed using the Frenet-Serret formula (Eq. (3)) [19].

$$
\begin{aligned}
& \boldsymbol{t}=\frac{d \boldsymbol{r}(s)}{d s} \\
& \boldsymbol{n}=\frac{\frac{d^{2} \boldsymbol{r}(s)}{d s^{2}}}{\left\|\frac{d^{2} \boldsymbol{r}(s)}{d s^{2}}\right\|} \\
& \boldsymbol{b}=\boldsymbol{t} \times \boldsymbol{n} \\
& \kappa(s)=\left\|\frac{d^{2} \boldsymbol{r}(s)}{d s^{2}}\right\| \text { and } \tau(s)=\frac{d \boldsymbol{n}(s)}{d s} \cdot \boldsymbol{b}
\end{aligned}
$$


$\left[\begin{array}{l}\frac{d \boldsymbol{t}}{d s} \\ \frac{d \boldsymbol{n}}{d s} \\ \frac{d \boldsymbol{b}}{d s}\end{array}\right]=\left[\begin{array}{ccc}0 & \kappa & 0 \\ -\kappa & 0 & \tau \\ 0 & -\tau & 0\end{array}\right]\left[\begin{array}{l}\boldsymbol{t} \\ \boldsymbol{n} \\ \boldsymbol{b}\end{array}\right]$

Let $\boldsymbol{v}=v_{t} \boldsymbol{t}+v_{n} \boldsymbol{n}+v_{b} \boldsymbol{b}$ be a vector field represented in the Frenet-Serret frame. Using the chain rule, the derivative of this field with respect to the arc-length parameter of the curve can be expressed as

$\frac{d \boldsymbol{v}}{d s}=\left(\frac{d v_{t}}{d s}-\kappa v_{n}\right) \boldsymbol{t}+\left(\frac{d v_{n}}{d s}+\kappa v_{t}-\tau v_{b}\right) \boldsymbol{n}+\left(\frac{d v_{b}}{d s}+\tau v_{n}\right) \boldsymbol{b}$
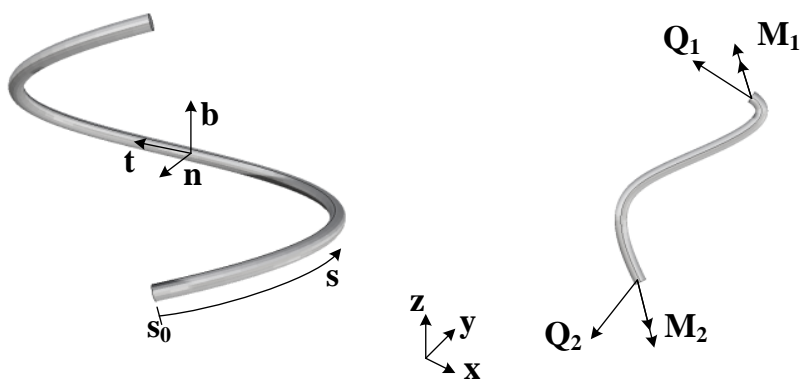

Figure 1: Curved beam - Frenet-Serret basis and beam section with internal forces.

\subsection{Equilibrium}

Figure 1 shows a curved beam with the global and local (Frenet-Serret) basis as well as a curved beam section under generic loading. Force equilibrium requires that $[13,20]$

$$
\boldsymbol{Q}_{2}-\boldsymbol{Q}_{1}+\int_{s_{1}}^{s_{2}} \boldsymbol{q}(s) d s=\mathbf{0}
$$

where $\boldsymbol{Q}_{1}$ and $\boldsymbol{Q}_{2}$ are vectors of reaction forces at ends 1 and 2 of the beam (Figure 1) and $\boldsymbol{q}(s)=$ $q_{t}(s) \boldsymbol{t}+q_{n}(s) \boldsymbol{n}+q_{b}(s) \boldsymbol{b}$ is the vector of distributed forces acting on the beam.

Moment equilibrium requires that $[13,20]$

$$
\boldsymbol{M}_{2}-\boldsymbol{M}_{1}+\left(\boldsymbol{r}_{2} \times \boldsymbol{Q}_{2}\right)-\left(\boldsymbol{r}_{1} \times \boldsymbol{Q}_{1}\right)+\int_{s_{1}}^{s_{2}}[\boldsymbol{m}(s)+\boldsymbol{r}(s) \times \boldsymbol{q}(s)] d s=\mathbf{0}
$$


where $\boldsymbol{r}_{1}$ and $\boldsymbol{r}_{2}$ are the distances from the origin to the two end points, $\boldsymbol{M}_{1}$ and $\boldsymbol{M}_{2}$ are vectors of reaction moments and $\boldsymbol{m}(s)=m_{t}(s) \boldsymbol{t}+m_{n}(s) \boldsymbol{n}+m_{b}(s) \boldsymbol{b}$ is the vector of distributed moments acting on the beam. Equations (5) and (6) can be rewritten as:

$$
\begin{aligned}
& \int_{s_{1}}^{s_{2}}\left[\frac{d \boldsymbol{Q}}{d s}+\boldsymbol{q}(s)\right] d s=\mathbf{0} \\
& \int_{s_{1}}^{s_{2}}\left[\frac{d \boldsymbol{M}}{d s}+\frac{d(\boldsymbol{r} \times \boldsymbol{Q})}{d s}+\boldsymbol{m}(s)+\boldsymbol{r}(s) \times \boldsymbol{q}(s)\right] d s=\mathbf{0}
\end{aligned}
$$

where $\boldsymbol{Q}=\left[Q_{t}, Q_{n}, Q_{b}\right]^{T}$ and $\boldsymbol{M}=\left[M_{t}, M_{n}, M_{b}\right]^{T}$ are the force and moment fields. Since equations

(7) hold for arbitrary arc-length, this implies that

$$
\begin{aligned}
& \frac{d \boldsymbol{Q}}{d s}+\boldsymbol{q}(s)=\mathbf{0} \\
& \frac{d \boldsymbol{M}}{d s}+\frac{d(\boldsymbol{r} \times \boldsymbol{Q})}{d s}+\boldsymbol{m}(s)+\boldsymbol{r}(s) \times \boldsymbol{q}(s)=\mathbf{0}
\end{aligned}
$$

Finally, using the result that $\boldsymbol{t}=\frac{d \boldsymbol{r}}{d s}$ and $\boldsymbol{r} \times \frac{d \boldsymbol{Q}}{d s}=-\boldsymbol{r} \times \boldsymbol{q}(s)($ Eq. (8) $)$, the equilibrium equations are simplified as

$$
\begin{aligned}
& \frac{d \boldsymbol{Q}}{d s}+\boldsymbol{q}(s)=\mathbf{0} \\
& \frac{d \boldsymbol{M}}{d s}+\boldsymbol{t} \times \boldsymbol{Q}+\boldsymbol{m}(s)=\mathbf{0}
\end{aligned}
$$

\subsection{Kinematics}

The beam kinematics for linear theory is defined by the displacement of the centroidal curve and rotation of the beam cross-section at each point of the centroidal curve. The displacement vector is represented in the Frenet-Serret basis by the vector field $\boldsymbol{u}=u_{t} \boldsymbol{t}+u_{n} \boldsymbol{n}+u_{b} \boldsymbol{b}$ and the rotation vector is represented by $\boldsymbol{\theta}=\theta_{t} \boldsymbol{t}+\theta_{n} \boldsymbol{n}+\theta_{b} \boldsymbol{b}$. It is assumed that plane sections normal to the line of centroids remain plane after deformation; however, they are not required to remain normal to the line of centroids after deformation, thus allowing for shear deformations. Generalized strain measures: $\boldsymbol{\varepsilon}(s)$, representing axial and shear strains, and $\boldsymbol{\beta}(s)$, representing twisting and flexural 
strains, can be obtained by using the fact that under rigid body motion the strain disappears [13].

The generalized strain measures are given by $[13,20]$

$$
\begin{aligned}
& \boldsymbol{\beta}(s)=\frac{d \boldsymbol{\theta}}{d s} \\
& \boldsymbol{\varepsilon}(s)=\frac{d \boldsymbol{u}}{d s}-\boldsymbol{\theta} \times \boldsymbol{t}
\end{aligned}
$$

where $\boldsymbol{\varepsilon}=\left[\varepsilon_{t}, \varepsilon_{n}, \varepsilon_{b}\right]^{T}$ are the axial $\left(\varepsilon_{n}\right)$ and two shear $\left(\varepsilon_{n}\right.$ and $\left.\varepsilon_{b}\right)$ strains, and $\boldsymbol{\beta}=\left[\beta_{t}, \beta_{n}, \beta_{b}\right]^{T}$ are the twisting $\left(\beta_{t}\right)$ and two bending $\left(\beta_{n}\right.$ and $\left.\beta_{b}\right)$ strains along the respective axes.

\subsection{Constitutive Relations}

Linear isotropic homogeneous behavior is considered in this study, and for this case the constitutive relationships can be expressed as

$$
\begin{aligned}
& {\left[\begin{array}{l}
Q_{t} \\
Q_{n} \\
Q_{b}
\end{array}\right]=\left[\begin{array}{ccc}
E A & 0 & 0 \\
0 & G A_{n} & 0 \\
0 & 0 & G A_{b}
\end{array}\right]\left[\begin{array}{l}
\varepsilon_{t} \\
\varepsilon_{n} \\
\varepsilon_{b}
\end{array}\right]} \\
& {\left[\begin{array}{l}
M_{t} \\
M_{n} \\
M_{b}
\end{array}\right]=\left[\begin{array}{ccc}
G I_{t} & 0 & 0 \\
0 & E I_{n} & 0 \\
0 & 0 & E I_{b}
\end{array}\right]\left[\begin{array}{l}
\beta_{t} \\
\beta_{n} \\
\beta_{b}
\end{array}\right]}
\end{aligned}
$$

where $E$ is the modulus of elasticity, $A, A_{n}$ and $A_{b}$ are the total area and shear areas of the cross section, $G$ is the shear modulus, $I_{t}$ is the torsional constant, and $I_{n}$ and $I_{b}$ are the moments of inertia of the cross section with respect to $\boldsymbol{n}$ and $\boldsymbol{b}$ axes, respectively. Combining Eqs. (9), (10) and (11) gives the six equilibrium equations in terms of the unknown displacement and rotation fields

$$
\begin{aligned}
& \frac{d}{d s}\left[E A\left(\frac{d u_{t}}{d s}-\kappa u_{n}\right)\right]-\kappa G A_{n}\left(\frac{d u_{n}}{d s}+\kappa u_{t}-\tau u_{b}-\theta_{b}\right)+q_{t}=0 \\
& \frac{d}{d s}\left[G A_{n}\left(\frac{d u_{n}}{d s}+\kappa u_{t}-\tau u_{b}-\theta_{b}\right)\right]+\kappa E A\left(\frac{d u_{t}}{d s}-\kappa u_{n}\right)-\tau G A_{b}\left(\frac{d u_{b}}{d s}+\tau u_{n}+\theta_{n}\right)+q_{n}=0 \\
& \frac{d}{d s}\left[G A_{b}\left(\frac{d u_{b}}{d s}+\tau u_{n}+\theta_{n}\right)\right]+\tau G A_{n}\left(\frac{d u_{n}}{d s}+\kappa u_{t}-\tau u_{b}-\theta_{b}\right)+q_{b}=0 \\
& \frac{d}{d s}\left[G I_{t}\left(\frac{d \theta_{t}}{d s}-\kappa \theta_{n}\right)\right]-\kappa E I_{n}\left(\frac{d \theta_{n}}{d s}-\tau \theta_{b}+\kappa \theta_{t}\right)+m_{t}=0
\end{aligned}
$$




$$
\begin{aligned}
& \frac{d}{d s}\left[E I_{n}\left(\frac{d \theta_{n}}{d s}-\tau \theta_{b}+\kappa \theta_{t}\right)\right]+\kappa G I_{t}\left(\frac{d \theta_{t}}{d s}-\kappa \theta_{n}\right)-G A_{b}\left(\frac{d u_{b}}{d s}+\tau u_{n}+\theta_{n}\right) \\
& -\tau E I_{b}\left(\frac{d \theta_{b}}{d s}+\tau \theta_{n}\right)+m_{n}=0 \\
& \frac{d}{d s}\left[E I_{b}\left(\frac{d \theta_{b}}{d s}+\tau \theta_{n}\right)\right]+G A_{n}\left(\frac{d u_{n}}{d s}+\kappa u_{t}-\tau u_{b}-\theta_{b}\right)+\tau E I_{n}\left(\frac{d \theta_{n}}{d s}-\tau \theta_{b}+\kappa \theta_{t}\right)+m_{b}=0
\end{aligned}
$$

Equations (12) are the strong form of the equilibrium conditions in the local Frenet-Serret frame. In this study, the finite element method based on isogeometric analysis is presented to numerically solve the above system of equations.

\section{NURBS curves and isogeometric analysis}

\subsection{NURBS curves}

A non-uniform rational B-spline (NURBS) curve is represented by a linear combination of NURBS basis functions as

$$
\boldsymbol{x}(\xi)=\sum_{q=1}^{n} R_{q, p}(\xi) \boldsymbol{x}_{q}
$$

where $\xi \in\left[\xi_{1}, \xi_{n+p+1}\right]$ is the scalar that is used to parameterize the curve, $\boldsymbol{x}(\xi)=$ $[x(\xi), y(\xi), z(\xi)]$ is the position vector of a point on the NURBS curve, $R_{q, p}(\xi)$ is the NURBS basis function given by $[2,3]$

$$
R_{q, p}(\xi)=\frac{N_{q, p}(\xi) w_{q}}{\sum_{j=1}^{n} N_{j, p}(\xi) w_{j}}
$$

where $N_{q, p}(\xi)$ is the B-spline basis function of degree $p$. A B-spline patch $\mathcal{P}$ is defined by a knot vector $\Xi=\left\{\xi_{1}, \xi_{2}, \ldots, \xi_{n+p+1}\right\}$ with $n+p+1$ knots, $\xi_{k}$, in which the knots are arranged in a non-decreasing order, i.e. $\xi_{k} \leq \xi_{k+1}$. The basis functions $N_{q, p}(\xi)$ can be recursively obtained as follows $[2,21,22]$ 


$$
\begin{aligned}
& N_{q, 0}(\xi)=\left\{\begin{array}{cc}
1 & \text { if } \xi_{q} \leq \xi<\xi_{q+1} \\
0 & \text { otherwise }
\end{array}, \quad p=0\right. \\
& N_{q, p}(\xi)=\frac{\xi-\xi_{q}}{\xi_{q+p}-\xi_{q}} N_{q, p-1}(\xi)+\frac{\xi_{q+p+1}-\xi}{\xi_{q+p+1}-\xi_{q+1}} N_{q+1, p-1}(\xi), \quad p \geq 1
\end{aligned}
$$

An example of a NURBS curve is shown in Figure 2, where Figure 2(a) shows the NURBS basis functions $\left\{R_{q, p}(\xi)\right\}_{q=1}^{10}$ based on an open knot vector. Open implies that the multiplicity of the first $\left(\xi_{1}=0\right)$ and the last $\operatorname{knot}\left(\xi_{14}=7\right)$ values is equal to $p+1=4$; the starting and ending control points are interpolated when an open knot vector is used [2]. Figure 2(b) shows the NURBS curve constructed by using the control points $\boldsymbol{x}_{q}(q=1, \ldots, 10)$ and the basis $\left\{R_{q, p}(\xi)\right\}_{q=1}^{10}$. In general, the continuity of the NURBS curve within each non-zero knot span is $C^{\infty}$, while the continuity across an interior knot is $C^{p-m_{k}}$, where $m_{k}$ is the multiplicity of the interior knot. In Figure 2(a), the continuity of the whole patch/curve is $C^{2}$. The NURBS curve shown in Figure 2(b) is constructed by using one patch; however, multiple patches can also be used to build more complex structural geometries.

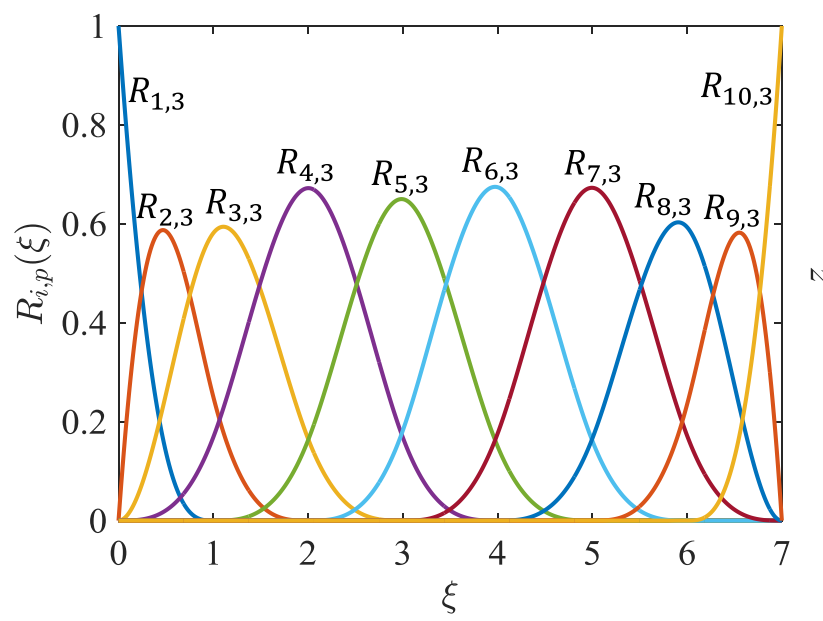

(a) NURBS basis functions

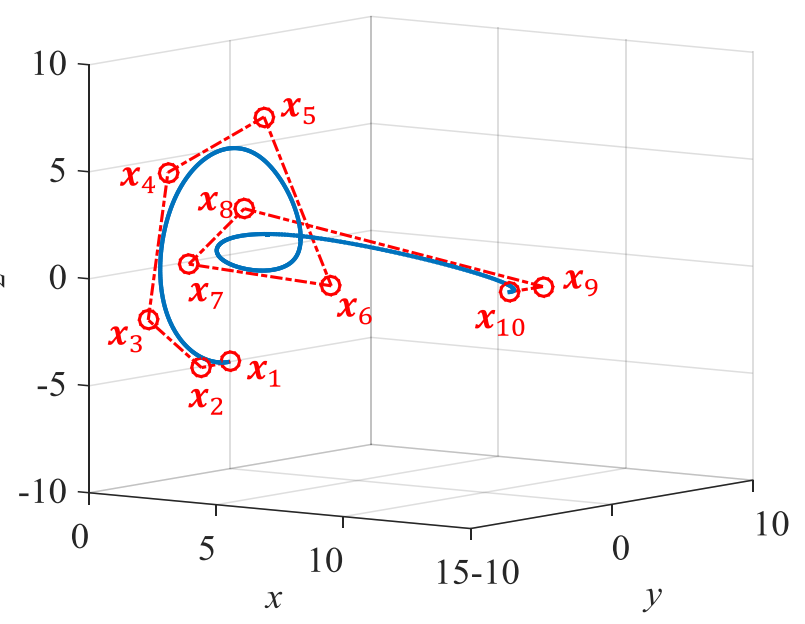

(b) NURBS curve

Figure 2: NURBS basis functions and NURBS curve with control points $\left\{\boldsymbol{x}_{1}, \ldots, \boldsymbol{x}_{10}\right\}$ based on an open knot vector $\boldsymbol{\Xi}=\{0,0,0,0,1,2,3,4,5,6,7,7,7,7\}$ 


\subsection{Isogeometric analysis (IGA)}

In isogeometric analysis (IGA) both the geometry and the unknown fields of interest displacement $\left(u_{t}, u_{n}\right.$ and $\left.u_{b}\right)$ and rotations $\left(\theta_{t}, \theta_{n}\right.$ and $\left.\theta_{b}\right)$ in this case - are interpolated using the NURBS basis. In IGA, an "element" is defined as a non-zero knot span for numerical integration purposes since the basis functions are $C^{\infty}$ rational polynomials within each non-zero knot span; for example, there are seven elements in the patch defined by knot vector $\Xi$ in Figure 2 . To effectively use the architecture of existing finite element codes, Borden et al. [23] proposed the Bézier extraction operator to construct the basis functions. This method provides an element-wise structure to IGA that allows the implementation of IGA into existing FEA codes without changing subroutines such as assembly, data storing or processing algorithms. In this study, Bézier extraction operators are used to construct the shape functions and the shape function derivatives; further details about the Bézier extraction can be found in Borden et al. [23].

\section{Finite Element Formulation}

\subsection{Weak forms}

The weak forms are obtained by multiplying the six equations given in Eq. (12) by suitable test functions $-\delta u_{t}, \delta u_{n}, \delta u_{b}, \delta \theta_{t}, \delta \theta_{n}$, and $\delta \theta_{b}$ - and performing integration by parts as

$$
\begin{aligned}
& \int_{L}\left[\frac{d \delta u_{t}}{d s} E A \varepsilon_{t}+\delta u_{t} \kappa G A_{n} \varepsilon_{n}\right] d s=\int_{L} \delta u_{t} q_{t} d s+\left[\delta u_{t} E A \varepsilon_{t}\right]_{\Gamma} \\
& \int_{L}\left[\frac{d \delta u_{n}}{d s} G A_{n} \varepsilon_{n}-\delta u_{n} \kappa E A \varepsilon_{t}+\delta u_{n} \tau G A_{b} \varepsilon_{b}\right] d s=\int_{L} \delta u_{n} q_{n} d s+\left[\delta u_{n} G A_{n} \varepsilon_{n}\right]_{\Gamma} \\
& \int_{L}\left[\frac{d \delta u_{b}}{d s} G A_{b} \varepsilon_{b}-\delta u_{b} \tau G A_{n} \varepsilon_{n}\right] d s=\int_{L} \delta u_{b} q_{b} d s+\left[\delta u_{b} G A_{b} \varepsilon_{b}\right]_{\Gamma} \\
& \int_{L}\left[\frac{d \delta \theta_{t}}{d s} G I_{t} \beta_{t}+\delta \theta_{t} \kappa E I_{n} \beta_{n}\right] d s=\int_{L} \delta \theta_{t} m_{t} d s+\left[\delta \theta_{t} G I_{t} \beta_{t}\right]_{\Gamma}
\end{aligned}
$$




$$
\begin{aligned}
& \int_{L}\left[\frac{d \delta \theta_{n}}{d s} E I_{n} \beta_{n}-\delta \theta_{n} \kappa G I_{t} \beta_{t}+\delta \theta_{n} G A_{b} \varepsilon_{b}+\delta \theta_{n} \tau E I_{b} \beta_{b}\right] d s \\
& =\int_{L} \delta \theta_{n} m_{n} d s+\left[\delta \theta_{n} E I_{n} \beta_{n}\right]_{\Gamma} \\
& \int_{L}\left[\frac{d \delta \theta_{b}}{d s} E I_{b} \beta_{b}-\delta \theta_{b} G A_{n} \varepsilon_{n}-\delta \theta_{b} \tau E I_{n} \beta_{n}\right] d s=\int_{L} \delta \theta_{b} m_{b} d s+\left[\delta \theta_{b} E I_{b} \beta_{b}\right]_{\Gamma}
\end{aligned}
$$

where $[\mathbf{a}]_{\Gamma}$ represents the boundary terms.

\subsection{Discretization and IGA formulation}

In IGA, both geometry and solution space are spanned by NURBS basis. Consider a curved beam represented by a NURBS curve of degree $p$ with control points $\boldsymbol{x}_{q}$, weights $w_{q}$ and knot vector $\Xi=\left\{\xi_{1}, \xi_{2}, \ldots, \xi_{n+p+1}\right\}(q=1,2, \ldots, n)$. In isogeometric terminology, the beam is considered as a patch while the non-zero knot $\operatorname{span}\left[\xi_{q}, \xi_{q+1}\right]$ is considered as an element in which numerical quadrature is implemented. For example, Figure 3 shows the IGA based discretization of a 2-D curved semi-circular beam member with 7 control points $\left(\boldsymbol{x}_{1}\right.$ to $\left.\boldsymbol{x}_{7}\right)$ and 4 Bézier elements. Since second degree NURBS bases is used, the control point $\boldsymbol{x}_{4}$ is interpolated as the multiplicity of knot $\xi=0.5$ is 2 .

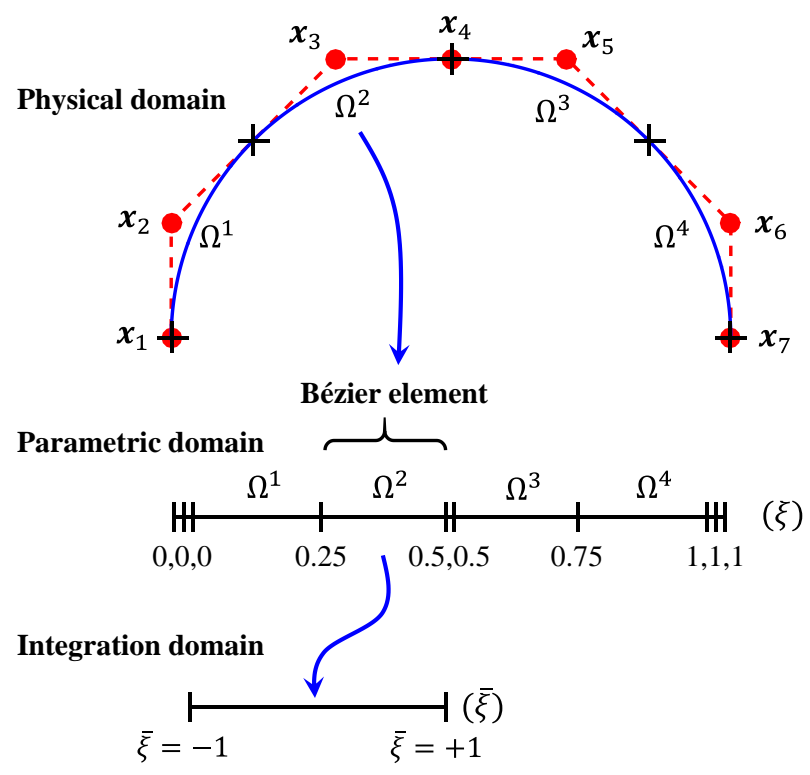


Figure 3: IGA discretization of a curved beam.

Within one element $\Omega^{e} \in\left[\xi_{q}, \xi_{q+1}\right]\left(\xi_{q} \neq \xi_{q+1}\right)$, the number of support control points and the corresponding interpolation functions is $p+1$ due to the local support property of B-spline basis, see Ref. [2] for further details. This means that only $p+1$ interpolation functions are non-zero within the element, and these $p+1$ interpolation functions can be used to span the geometry and solution spaces within the element. Using this local support property, geometry, displacement fields and their derivatives within the $i^{\text {th }}$ Bézier element can be expressed as follows

$$
\begin{aligned}
& \boldsymbol{x}^{h}(\xi)=\sum_{k=q_{i}-p}^{q_{i}} R_{k, p}(\xi) \boldsymbol{x}_{k}=\boldsymbol{N}^{e} \boldsymbol{x}^{e} \\
& \boldsymbol{u}^{h}(\xi)=\sum_{k=q_{i}-p}^{q_{i}} R_{k, p}(\xi) \boldsymbol{u}_{k}=\boldsymbol{N}^{e} \boldsymbol{u}^{e} \\
& \boldsymbol{\theta}^{h}(\xi)=\sum_{k=q_{i}-p}^{q_{i}} R_{k, p}(\xi) \boldsymbol{\theta}_{k}=\boldsymbol{N}^{e} \boldsymbol{\theta}^{e}
\end{aligned}
$$

where $\boldsymbol{x}^{h}(\xi)=[x, y, z]^{T}, \boldsymbol{u}^{h}(\xi)=\left[u_{t}, u_{n}, u_{b}\right]^{T}$ and $\boldsymbol{\theta}^{h}(\xi)=\left[\theta_{t}, \theta_{n}, \theta_{b}\right]^{T}$ are the coordinate vector, displacement vector and rotation vector, respectively, of a point on the Bézier element; $q_{i}$ the largest integer such that $\xi \geq \xi_{q_{i}} ; x_{q}=\left[x_{q}, y_{q}, z_{q}\right]^{T}$ is the coordinate vector of the $q^{\text {th }}$ control point; $\boldsymbol{u}_{q}=\left[u_{t}^{q}, u_{n}^{q}, u_{b}^{q}\right]^{T}$ and $\boldsymbol{\theta}_{q}=\left[\theta_{t}^{q}, \theta_{n}^{q}, \theta_{b}^{q}\right]^{T}$ are the displacement vector and rotation vector, respectively, of the $q^{\text {th }}$ control point; $\boldsymbol{N}^{e}$ is the shape function matrix and $\boldsymbol{x}^{e}, \boldsymbol{u}^{e}$ and $\boldsymbol{\theta}^{e}$ are the vector of coordinates and unknown displacement quantities at the control points for a Bézier element. Note that the interpolation of geometry is carried out in the global coordinate system; however, solution space is represented in the local Frenet-Serret frame (Eq. (1)). From the continuity requirement in the weak form, only $C^{0}$ continuity is needed (Eq. (16)). However, the continuity of the local Frenet-Serret frame should also be guaranteed which means at each point 
on the curve the Frenet-Serret frame should be uniquely defined. Galerkin method is used for constructing the finite element approximations, wherein both the unknown fields ( $\boldsymbol{u}$ and $\boldsymbol{\theta})$ and the corresponding weighting functions, $\delta \boldsymbol{u}=\left[\delta u_{t}, \delta u_{n}, \delta u_{b}\right]^{T}$ and $\delta \boldsymbol{\theta}=\left[\delta \theta_{t}, \delta \theta_{n}, \delta \theta_{b}\right]^{T}$, are approximated using the NURBS basis, i.e.

$$
\begin{gathered}
\delta \boldsymbol{u}^{h}(\xi)=\sum_{k=q_{i}-p}^{q_{i}} R_{k, p}(\xi) \delta \boldsymbol{u}_{k}=\boldsymbol{N}^{e} \delta \boldsymbol{u}^{e} \\
\delta \boldsymbol{\theta}^{h}(\xi)=\sum_{k=q_{i}-p}^{q_{i}} R_{k, p}(\xi) \delta \boldsymbol{\theta}_{k}=\boldsymbol{N}^{e} \delta \boldsymbol{\theta}^{e}
\end{gathered}
$$

The shape function derivatives are calculated using the chain rule as

$$
\begin{aligned}
& \frac{d(\mathbf{\square})}{d s}=\frac{d(\mathbf{\square})}{d \xi} \frac{1}{J^{h}} \\
& g^{h}(\xi)=\frac{d s}{d \xi}=\sqrt{\left(\frac{d x^{h}}{d \xi}\right)^{2}+\left(\frac{d y^{h}}{d \xi}\right)^{2}+\left(\frac{d z^{h}}{d \xi}\right)^{2}} \\
& \frac{d x^{h}}{d \xi}=\sum_{k=q_{i}-p}^{q_{i}} \frac{d R_{k, p}(\xi)}{d \xi} x_{k}, \quad \frac{d y^{h}}{d \xi}=\sum_{k=q_{i}-p}^{q_{i}} \frac{d R_{k, p}(\xi)}{d \xi} y_{k}, \\
& \frac{d z^{h}}{d \xi}=\sum_{k=q_{i}-p}^{q_{i}} \frac{d R_{k, p}(\xi)}{d \xi} z_{k}
\end{aligned}
$$

By taking the derivatives of NURBS shape functions, the strains within a Bézier element are expressed as

$$
\begin{aligned}
\varepsilon_{t}^{h}(\xi) & =\boldsymbol{B}^{e} \boldsymbol{u}_{t}^{e}-\kappa \boldsymbol{N}^{e} \boldsymbol{u}_{n}^{e} \\
\varepsilon_{n}^{h}(\xi) & =\boldsymbol{B}^{e} \boldsymbol{u}_{n}^{e}+\kappa \boldsymbol{N}^{e} \boldsymbol{u}_{t}^{e}-\tau \boldsymbol{N}^{e} \boldsymbol{u}_{b}^{e}-\boldsymbol{N}^{e} \boldsymbol{\theta}_{b}^{e} \\
\varepsilon_{b}^{h}(\xi) & =\boldsymbol{B}^{e} \boldsymbol{u}_{b}^{e}+\tau \boldsymbol{N}^{e} \boldsymbol{u}_{n}^{e}+\boldsymbol{N}^{e} \boldsymbol{\theta}_{n}^{e} \\
\beta_{t}^{h}(\xi) & =\boldsymbol{B}^{e} \boldsymbol{\theta}_{t}^{e}-\kappa \boldsymbol{N}^{e} \boldsymbol{\theta}_{n}^{e} \\
\beta_{n}^{h}(\xi) & =\boldsymbol{B}^{e} \boldsymbol{\theta}_{n}^{e}-\tau \boldsymbol{N}^{e} \boldsymbol{\theta}_{b}^{e}+\kappa \boldsymbol{N}^{e} \boldsymbol{\theta}_{t}^{e} \\
\beta_{b}^{h}(\xi) & =\boldsymbol{B}^{e} \boldsymbol{\theta}_{b}^{e}+\tau \boldsymbol{N}^{e} \boldsymbol{\theta}_{n}^{e}
\end{aligned}
$$


where $\boldsymbol{B}^{e}$ is the shape function derivative matrix. Moreover, curvature and torsion are calculated from the parametric NURBS representation of the geometry as

$$
\begin{gathered}
\kappa(\xi)=\frac{\left\|\boldsymbol{x}^{\prime} \times \boldsymbol{x}^{\prime \prime}\right\|}{\left\|\boldsymbol{x}^{\prime}\right\|^{3}} \\
\tau(\xi)=\frac{\left(\boldsymbol{x}^{\prime} \times \boldsymbol{x}^{\prime \prime}\right) \cdot \boldsymbol{x}^{\prime \prime \prime}}{\left\|\boldsymbol{x}^{\prime} \times \boldsymbol{x}^{\prime \prime}\right\|^{2}}
\end{gathered}
$$

where the prime indicates the derivative with respect to the parameter $\xi$. Finally, applying the IGA interpolations described above to weak forms (Eq. (16)), the equilibrium equations are expressed in residual forms as

$$
\begin{aligned}
& \boldsymbol{R}(\boldsymbol{d})=\boldsymbol{F}_{\text {int }}(\boldsymbol{d})-\boldsymbol{F}_{\text {ext }}=\mathbf{0} \\
& \boldsymbol{F}_{\text {int }}(\boldsymbol{d})=\underset{e=1}{n_{\text {ele }}} \boldsymbol{F}_{\text {int }}^{e} \\
& \boldsymbol{F}_{\text {ext }}=\underset{e=1}{n_{\text {ele }}} \boldsymbol{F}_{\text {ext }}^{e}+\overline{\boldsymbol{F}}_{\text {ext }}
\end{aligned}
$$

where $\boldsymbol{R}(\boldsymbol{d})$ is the global residual vector, $\boldsymbol{F}_{\text {int }}$ is the internal force vector, $\boldsymbol{F}_{\text {ext }}$ is the external force vector, $\boldsymbol{F}_{i n t}^{e}$ is the internal force vector for a Bézier element, $\boldsymbol{F}_{e x t}^{e}$ is the external force vector for a Bézier element due to distributed loads, $\overline{\boldsymbol{F}}_{\text {ext }}$ is the external force contribution of the point loads, $\mathcal{A}$ is the standard finite element assembly operator, and $n_{\text {ele }}$ is the total number of Bézier element in the domain. The internal and external force vectors for a Bézier element are expressed as

$$
\boldsymbol{F}_{\text {int }}^{e}=\left[\begin{array}{c}
\boldsymbol{F}_{\text {int }, u_{t}}^{e} \\
\boldsymbol{F}_{\text {int }, u_{n}}^{e} \\
\boldsymbol{F}_{\text {int }, u_{b}}^{e} \\
\boldsymbol{F}_{\text {int }, \theta_{t}}^{e} \\
\boldsymbol{F}_{\text {int } \theta_{n}}^{e} \\
\boldsymbol{F}_{\text {int }, \theta_{b}}^{e}
\end{array}\right], \boldsymbol{F}_{\text {ext }}^{e}=\left[\begin{array}{c}
\boldsymbol{F}_{\text {ext }, u_{t}}^{e} \\
\boldsymbol{F}_{\text {ext, } u_{n}}^{e} \\
\boldsymbol{F}_{\text {ext, } u_{b}}^{e} \\
\boldsymbol{F}_{\text {ext, } \theta_{t}}^{e} \\
\boldsymbol{F}_{\text {ext }, \theta_{n}}^{e} \\
\boldsymbol{F}_{\text {ext }, \theta_{b}}^{e}
\end{array}\right]
$$

where

$$
\boldsymbol{F}_{\text {int, } u_{t}}^{e}=\int_{\Omega^{\mathrm{e}}}\left(\boldsymbol{B}^{\boldsymbol{e}^{T}} E A \varepsilon_{t}+\boldsymbol{N}^{e^{T}} \kappa G A_{n} \varepsilon_{n}\right) d s
$$




$$
\begin{aligned}
& \boldsymbol{F}_{i n t, u_{n}}^{e}=\int_{\Omega^{\mathrm{e}}}\left(\boldsymbol{B}^{e T} G A_{n} \varepsilon_{n}+\boldsymbol{N}^{e^{T}} \tau G A_{b} \varepsilon_{b}-\boldsymbol{N}^{e T} \kappa E A \varepsilon_{t}\right) d s \\
& \boldsymbol{F}_{\text {int, } u_{b}}^{e}=\int_{\Omega^{\mathrm{e}}}\left(\boldsymbol{B}^{e T} G A_{b} \varepsilon_{b}-\boldsymbol{N}^{e^{T}} \tau G A_{n} \varepsilon_{n}\right) d s \\
& \boldsymbol{F}_{i n t, \theta_{t}}^{e}=\int_{\Omega^{\mathrm{e}}}\left(\boldsymbol{B}^{\boldsymbol{e}^{T}} G I_{t} \beta_{t}+\boldsymbol{N}^{e T} \kappa E I_{n} \beta_{n}\right) d s \\
& \boldsymbol{F}_{i n t, \theta_{n}}^{e}=\int_{\Omega^{\mathrm{e}}}\left(\boldsymbol{B}^{e T} E I_{n} \beta_{n}-\boldsymbol{N}^{e^{T}} \kappa G I_{t} \beta_{t}+\boldsymbol{N}^{e T} G A_{b} \varepsilon_{b}+\boldsymbol{N}^{e T} \tau E I_{b} \beta_{b}\right) d s \\
& \boldsymbol{F}_{\text {int }, \theta_{b}}^{e}=\int_{\Omega^{\mathrm{e}}}\left(\boldsymbol{B}^{e^{T}} E I_{b} \beta_{b}-\boldsymbol{N}^{e^{T}} G A_{n} \varepsilon_{n}-\boldsymbol{N}^{e T} \tau E I_{n} \beta_{n}\right) d s \\
& \boldsymbol{F}_{\text {ext, } u_{t}}^{e}=\int_{\Omega^{\mathrm{e}}} \boldsymbol{N}^{e^{T}} q_{t} d s, \boldsymbol{F}_{\text {ext, } u_{n}}^{e}=\int_{\Omega^{\mathrm{e}}} \boldsymbol{N}^{e^{T}} q_{n} d s, \boldsymbol{F}_{\text {ext, } u_{b}}^{e}=\int_{\Omega^{\mathrm{e}}} \boldsymbol{N}^{e^{T}} q_{b} d s \\
& \boldsymbol{F}_{\text {ext, } \theta_{t}}^{e}=\int_{\Omega^{\mathrm{e}}} \boldsymbol{N}^{e T} m_{t} d s, \boldsymbol{F}_{\text {ext, } \theta_{n}}^{e}=\int_{\Omega^{\mathrm{e}}} \boldsymbol{N}^{\boldsymbol{e}^{T}} m_{n} d s, \boldsymbol{F}_{\text {ext, } \theta_{b}}^{e}=\int_{\Omega^{\mathrm{e}}} \boldsymbol{N}^{\boldsymbol{e}^{T}} m_{b} d s
\end{aligned}
$$

If the external distributed loads are defined in the global coordinate system, the loads can be transformed to the local Frenet-Serret system following

$$
\begin{aligned}
& {\left[\begin{array}{l}
q_{t} \\
q_{n} \\
q_{b}
\end{array}\right]=\left[\begin{array}{lll}
\mid & \mid & \mid \\
\boldsymbol{t} & \boldsymbol{n} & \boldsymbol{b} \\
\mid & \mid & \mid
\end{array}\right]^{T}\left[\begin{array}{l}
q_{x} \\
q_{y} \\
q_{z}
\end{array}\right]} \\
& {\left[\begin{array}{l}
m_{t} \\
m_{n} \\
m_{b}
\end{array}\right]=\left[\begin{array}{lll}
\mid & \mid & \mid \\
\boldsymbol{t} & \boldsymbol{n} & \boldsymbol{b} \\
\mid & \mid & \mid
\end{array}\right]^{T}\left[\begin{array}{l}
m_{x} \\
m_{y} \\
m_{z}
\end{array}\right]}
\end{aligned}
$$

where $\boldsymbol{t}, \boldsymbol{n}$ and $\boldsymbol{b}$ are the Frenet-Serret basis and are obtained from the parametric NURBS representation as

$$
t=\frac{x^{\prime}}{\left\|x^{\prime}\right\|}, \quad b=\frac{x^{\prime} \times x^{\prime \prime}}{\left\|x^{\prime} \times x^{\prime \prime}\right\|}, \quad n=b \times t
$$

For this linear system (Eq. (23)), the global stiffness matrix $(\boldsymbol{K})$ is assembled from the element stiffness matrices $\left(\boldsymbol{K}^{e}\right)$ as

$$
\boldsymbol{K}=\underset{e=1}{n_{\text {ele }}} \boldsymbol{K}^{e} \text { where }
$$




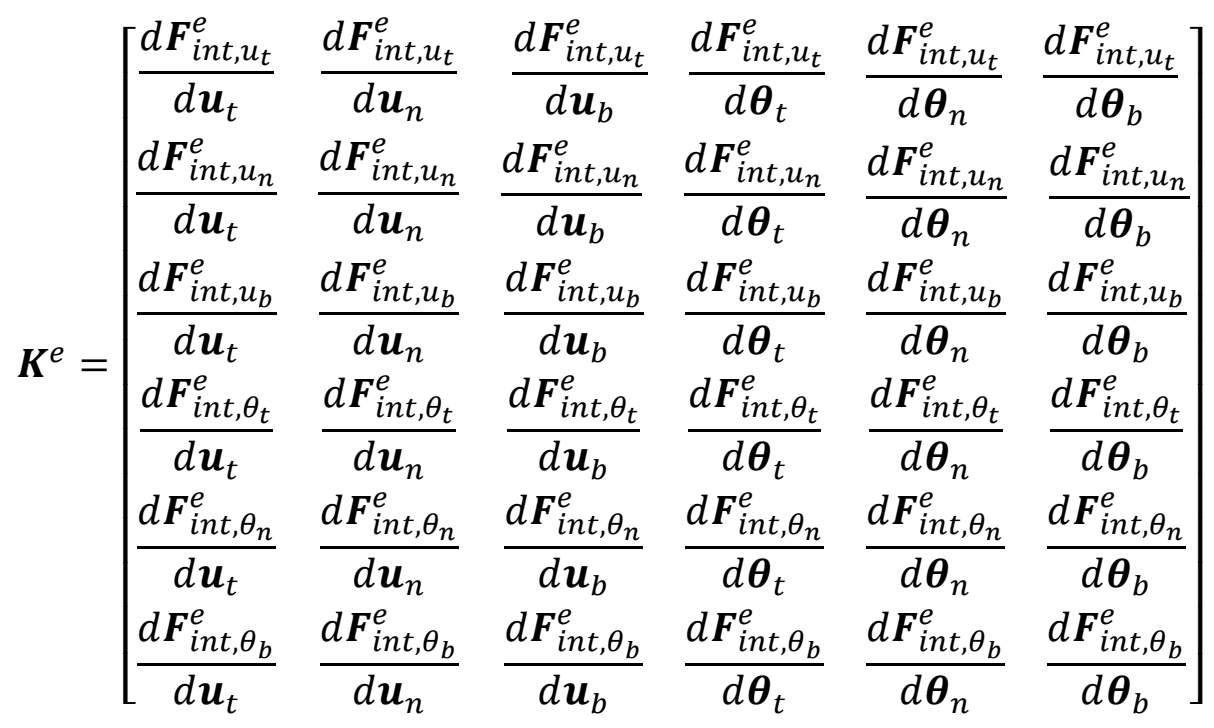

For the purpose of integration the Guass quadrature rule of appropriate order is used, and a typical integral over Bézier element $\Omega^{\mathrm{e}}=\left[\xi_{q}, \xi_{q+1}\right]$ is evaluated as

$$
\begin{gathered}
\int_{\Omega^{\mathrm{e}}} f(s) d s=\int_{\xi_{q}}^{\xi_{q+1}} f(\xi) \mathcal{J}^{h}(\xi) d \xi=\int_{-1}^{+1} f(\bar{\xi}) \mathcal{J}^{h}(\bar{\xi}) \frac{\xi_{q+1}-\xi_{q}}{2} d \bar{\xi} \\
=\sum_{r=1}^{n_{\text {int }}} f\left(\bar{\xi}_{r}\right) \mathcal{J}^{h}\left(\bar{\xi}_{r}\right) \frac{\xi_{q+1}-\xi_{q}}{2} \widehat{w}_{r}
\end{gathered}
$$

where $n_{\text {int }}=p+1$ is the number of integration points, $\bar{\xi}_{r}$ are the Gauss quadrature points and $\widehat{w}_{r}$ are the corresponding weights. The integral domain is transformed from parametric domain $\xi \in$ $\left[\xi_{q}, \xi_{q+1}\right]$ to parent element domain $\bar{\xi} \in[-1,1]$ through linear mapping: $\xi=\left(\frac{1-\bar{\xi}}{2}\right) \xi_{q}+$ $\left(\frac{1+\bar{\xi}}{2}\right) \xi_{q+1}$

\section{Example Problems}

The issue of locking is first investigated in the displacement based IGA formulation presented in Section 4, and then five example problems are considered to show the application of the proposed IGA approach. Geometries with different properties are included to show the effectiveness of the analysis technique. All analyses are carried out on a desktop computer with a Pentium $3.0 \mathrm{GHz}$ 
microprocessor using the Matlab ${ }^{\circledR}$ based finite element program CPSSL-FEA developed at the University of Notre Dame. All the NURBS geometries are created using the popular CAD software RHINO-3D [24], and the control point coordinates and weights are extracted from the IGES files. For all the examples following material properties are used: modulus of elasticity $E=200 \mathrm{GPa}$ and Poisson's ratio $v=0.3$.

\subsection{Locking}

Shear and membrane locking issues have been reported for the pure displacement field based finite element formulation in curved beams $[14,25]$. In the context of NURBS, several methodologies have been proposed to address the locking issue including selective reduced integration [18], discrete strain gap (DSG) method [26], and $\bar{B}$-projection method [18, 27], among others. Selective reduced integration is efficient and can remove locking in a $2^{\text {nd }}$ degree NURBS basis; however, there is no such general selective reduced integration rule that can handle locking for a higher order NURBS basis. The DSG method, originally proposed for straight beams, does not have good

accuracy in curved geometries [18]. While the $\bar{B}$-projection method, which is a special case of mixed methods, is shown to be robust in handling locking, it is computationally expensive as the sparsity of stiffness matrices is lost [18]. It is also important to note that a higher order NURBS basis can alleviate locking [26]. Before carrying out further analyses, the locking issue is first examined in the proposed NURBS based beam formulation.

A curved beam problem shown in Figure 4 is studied to investigate the effect of using a higher order NURBS basis in dealing with locking. The quarter circle beam of radius $R=5 \mathrm{~m}$ is fixed at one end and is subjected to uniform distributed moment $m_{z}=-0.01 \mathrm{~N} / \mathrm{m}$. The radius of the solid circular cross-section is determined by a dimensionless parameter $\varepsilon$ through 


$$
\varepsilon^{2}=\frac{I_{b}}{A L^{2}}
$$

where $A$ and $I_{b}$ are the area and moment of inertia of the cross section, respectively, and $L$ is the arc-length of the member. The exact solution to this problem is given by Eq. (31) and the relative $L^{2}$-error norm is shown in Figure 5 for different mesh size $h$. Clearly, as $\varepsilon^{2}$ decreases from $10^{-3}$ to $10^{-8}$, locking occurs as shown in Figure 5(a) for $2^{\text {nd }}$ degree NURBS basis. However, as shown in Figure 5(b) and Figure 5(c), with $3^{\text {rd }}$ and $4^{\text {th }}$ degree NURBS basis the locking is mitigated. In particular, when a cubic NURBS basis is used only slight locking appears (Figure 5b), while when a fourth degree basis is used there is no obvious locking (Figure 5c). Similar observations can be made for the convergence rates results for the moment field in Figure 6, wherein the higher order bases lead to better convergence rates with no obvious locking. This test case also validates the implementation of NURBS curved beams for the 2-D case. Considering the computational cost of the various methods in dealing with locking issues and the ease of implementing higher order NURBS basis, in the following examples, reasonable $\varepsilon^{2}$-values together with cubic or higher order NURBS basis are used to mitigate locking.

$$
u_{y}(s)=\frac{m_{z} R}{2 E I_{b}}\left(\left(2 R^{2}+\pi R s-s^{2}\right) \cos \left(\frac{s}{R}\right)+R\left(-2 R+(-\pi R+2 s) \sin \left(\frac{s}{R}\right)\right)\right)
$$

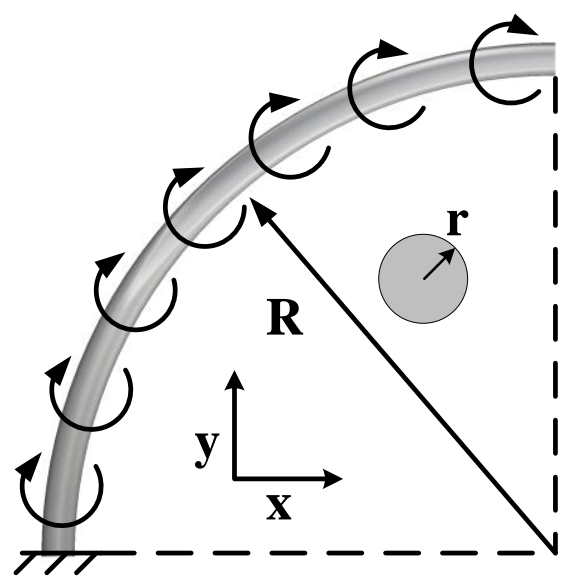

Figure 4: Curved cantilever - geometry and loading. 


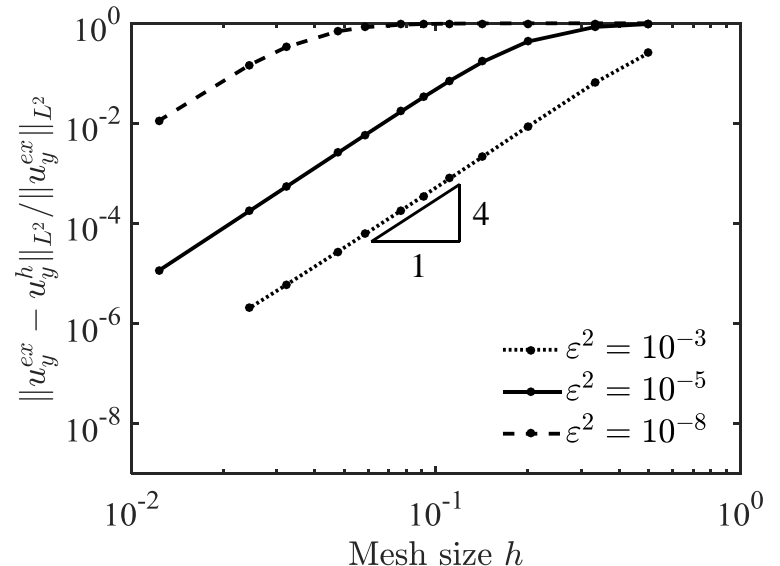

a) $2^{\text {nd }}$ degree

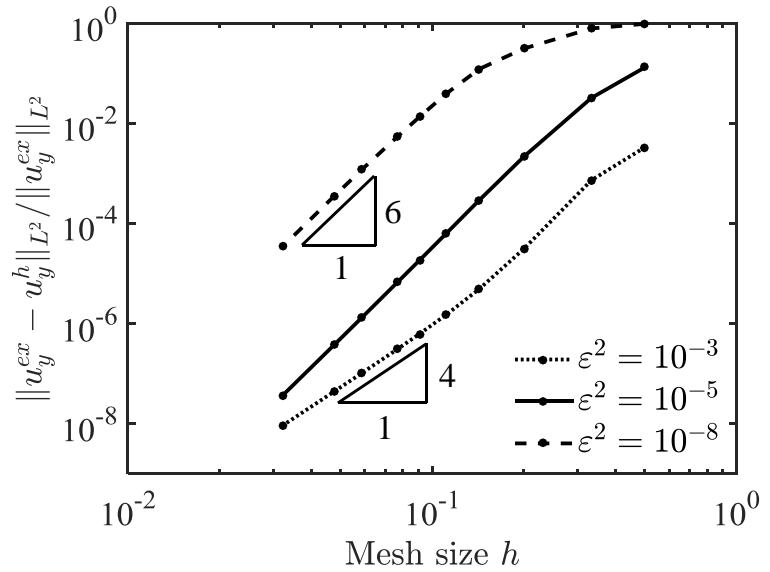

b) $3^{\text {rd }}$ degree

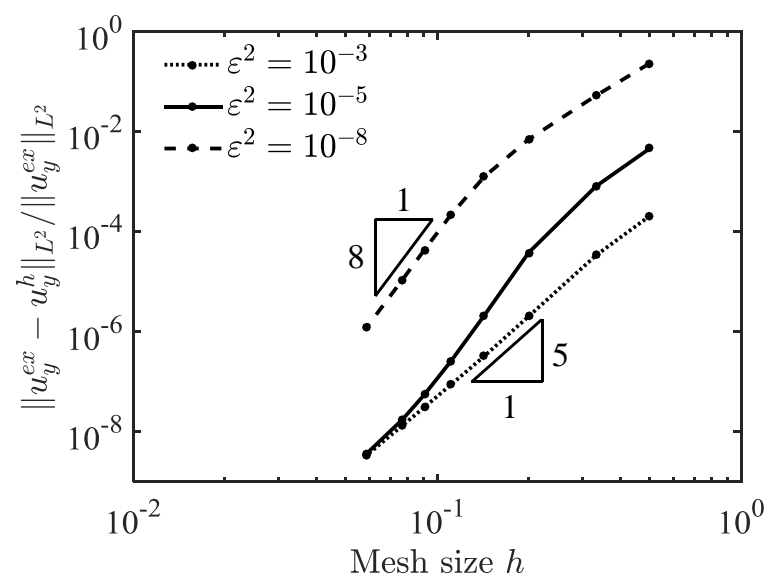

c) $4^{\text {th }}$ degree

Figure 5: Locking study - convergence rates of displacement field for $2^{\text {nd }}, 3^{\text {rd }}$ and $4^{\text {th }}$ degree NURBS basis.

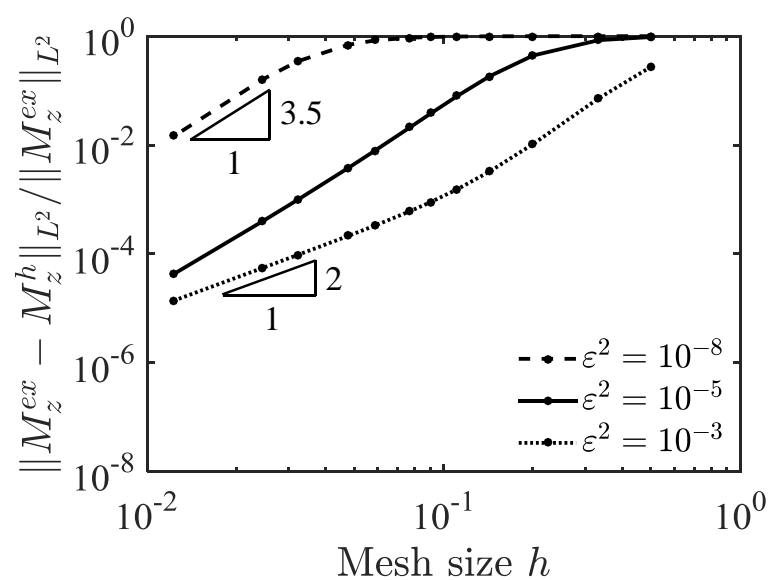

a) $2^{\text {nd }}$ degree

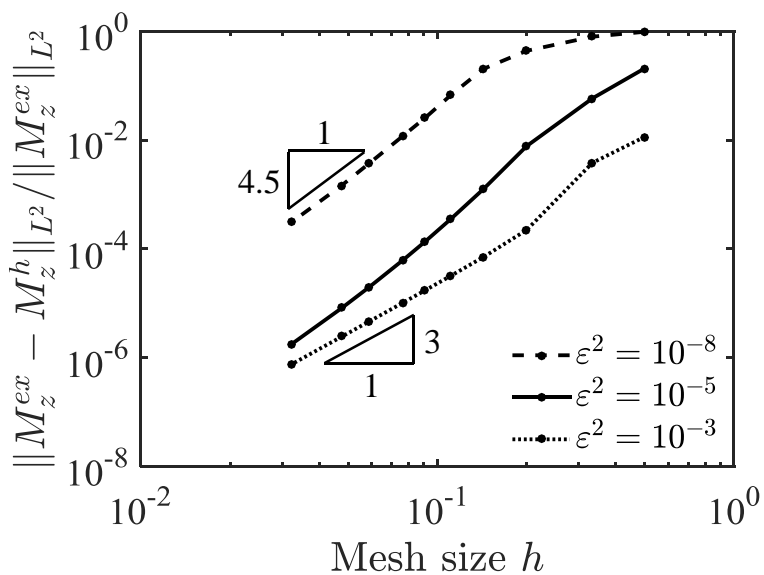

b) $3^{\text {rd }}$ degree 


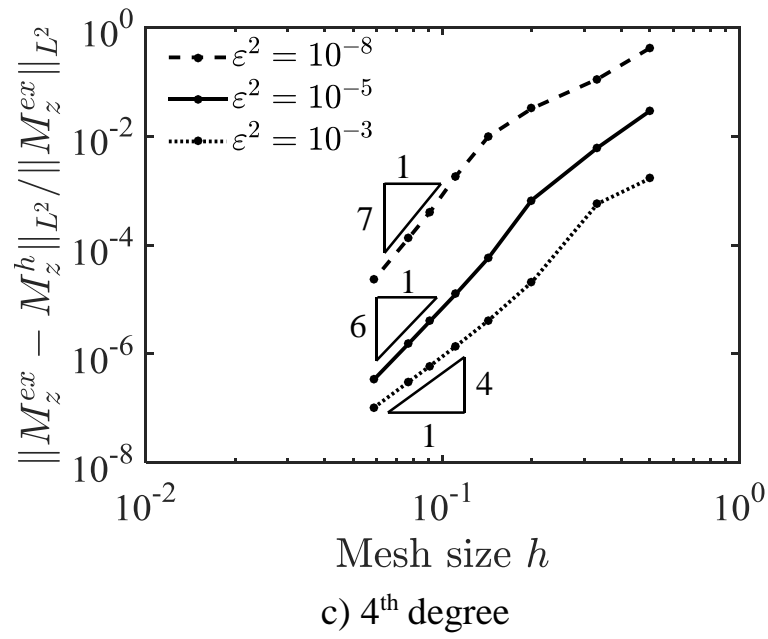

Figure 6: Locking study - convergence rates of moment field for $2^{\text {nd }}, 3^{\text {rd }}$ and $4^{\text {th }}$ degree NURBS basis.

\subsection{Circular Balcony}

The first example problem consists of a semi-circular curved 3-D balcony. Because of the geometry of this problem, the curvature is constant along the arc-length and is equal to $\kappa=1 / R$, where $R$ is the radius of the balcony. The geometry and loading are shown in Figure 7. The balcony has a radius $R=3 \mathrm{~m}$ and a square cross-section with $h=b=0.3 \mathrm{~m}$ is used and $\varepsilon^{2}=8.44 \times 10^{-5}$. The applied distributed load in the global ( - ) $z$ direction is $q=5 \mathrm{kN} / \mathrm{m}$ and the beam is fully fixed at both ends. An IGA mesh consisting of 16 elements with $4^{\text {th }}$ degree basis functions is used to perform the analysis. The nonzero internal forces and moments along the arc-length are calculated in the local coordinate system and plotted in Figure 8 while the nonzero displacements and rotations along the arc-length in terms of the local coordinate system are shown in Figure 9. Figure 10 shows the reference shape (red dotted line) and the deformed shape (blue solid line) with a magnification factor of 100 . 


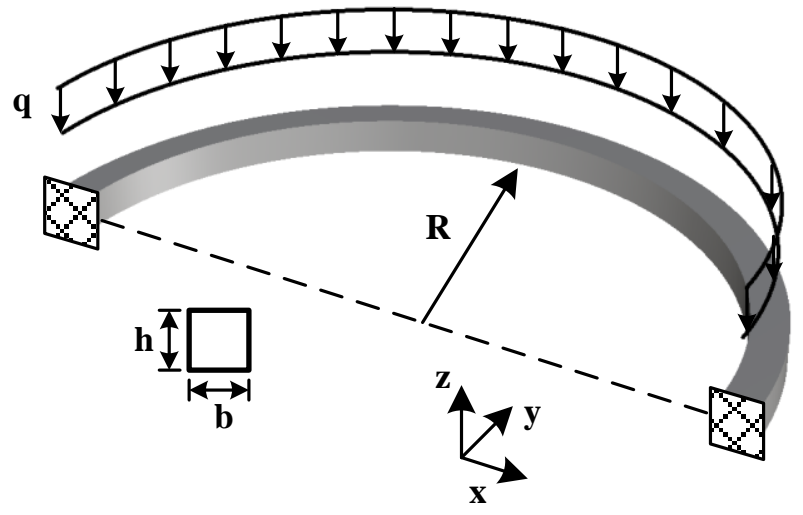

Figure 7: Circular balcony - geometry and loading.

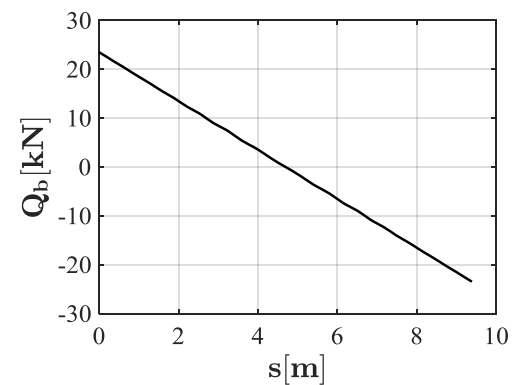

a) Shear force in b-direction

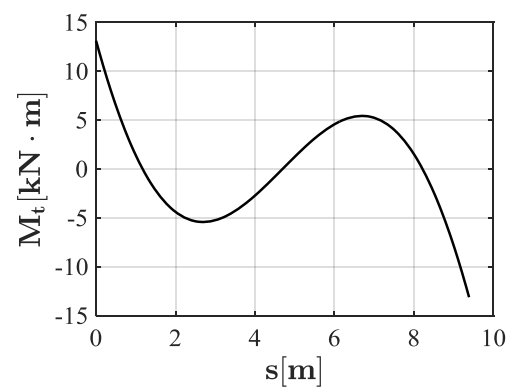

b) Moment about t-axis

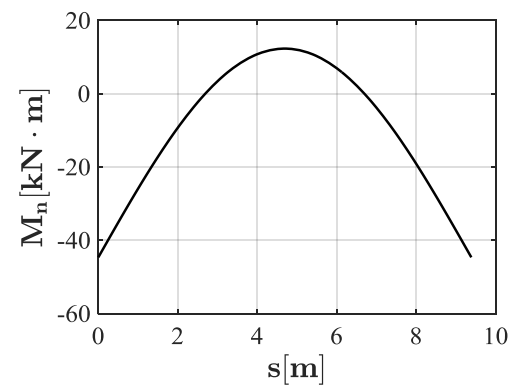

c) Moment about n-axis

Figure 8: Circular balcony - internal forces and moments along arc-length in local coordinate system.

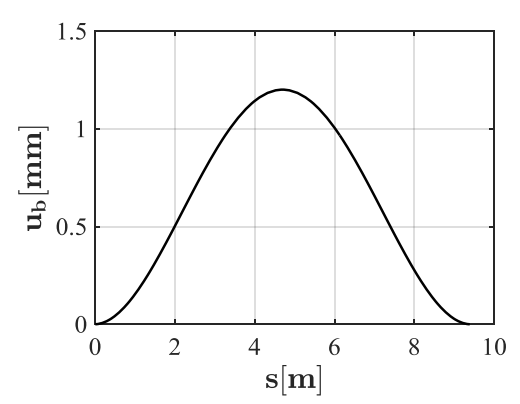

a) Displacement in b-direction

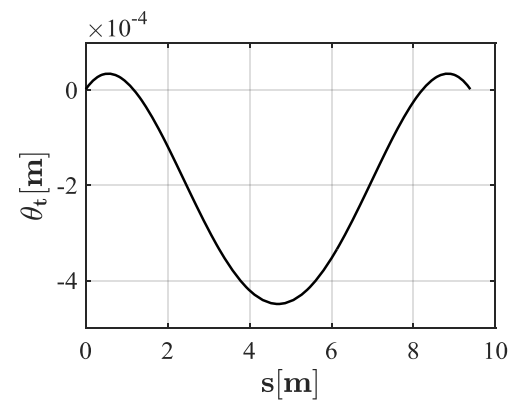

b) Rotation about t-axis

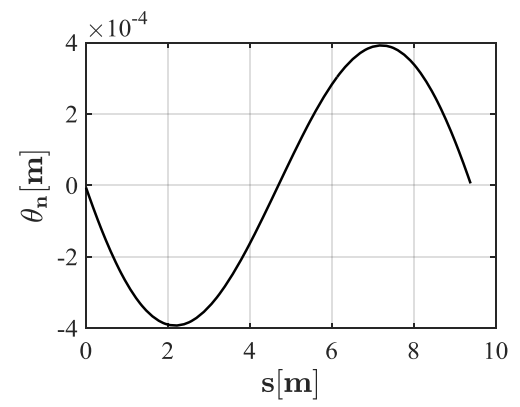

c) Rotation about n-axis

Figure 9: Circular balcony - displacements and rotations along arc-length in local coordinate system. 


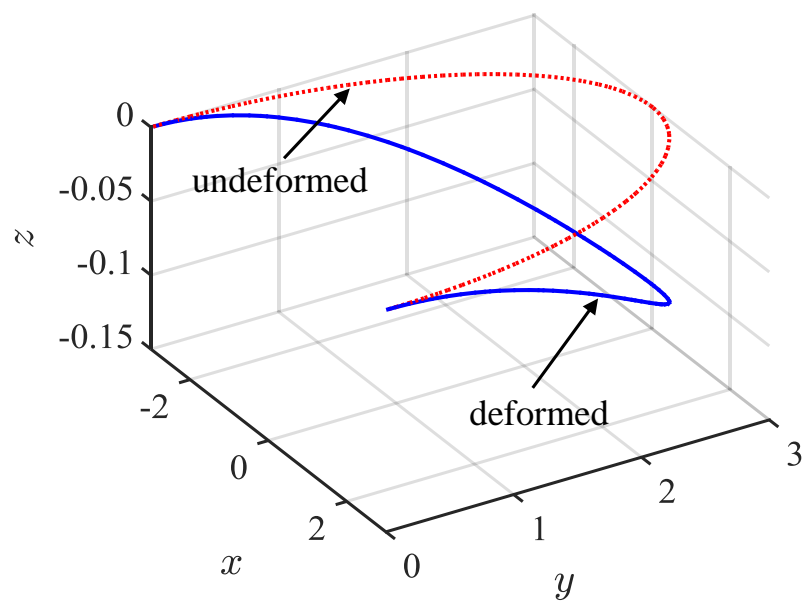

Figure 10: Circular balcony - reference and deformed shape.

\subsection{Logarithmic spiral}

The next example consists of a planar logarithmic spiral parameterized by the following equations:

$$
\boldsymbol{r}(s)=\{a \cos (s) \exp (b s), a \sin (s) \exp (b s), 0\}
$$

where $a$ and $b$ are constants. The values of $a$ and $b$ used are 2.41 and 0.405 , respectively. This leads to an overall height of 5.94 meters and width of 3.34 meters. The geometry and loading of this spiral structure are shown in Figure 11. The applied moment $M$ at the top end is $10 \mathrm{kN} \cdot \mathrm{m}$ and the bottom end is fully fixed. The cross-section used is a hollow circular section with an outer radius $r=15 \mathrm{~cm}$, thickness $t=4 \mathrm{~cm}$ and $\varepsilon^{2}=6.12 \times 10^{-5}$. This structural geometry has a nonconstant curvature along the length of the member and thus requires more control points to represent the geometry. Analysis is carried out using a $3^{\text {rd }}$ degree NURBS basis with 194 Bezier elements. Because of the applied loading conditions, the internal moment about the local $\boldsymbol{b}$-axis is a constant $10 \mathrm{kN} \cdot \mathrm{m}$ and all other internal forces are zero. The displacements and rotation along the arc-length in the local coordinate system are shown in Figure 12. The reference (red dotted line) and deformed (blue solid line) shapes are given in Figure 13 with a magnification factor of 100 used to show the deformed shape. 


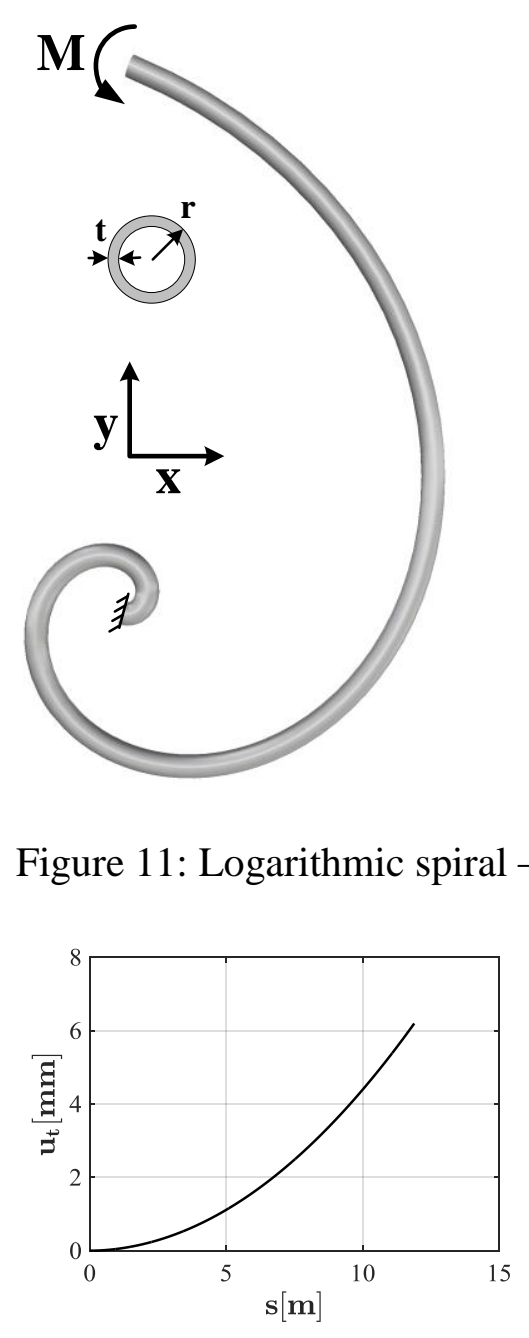

a) Displacement in t-direction

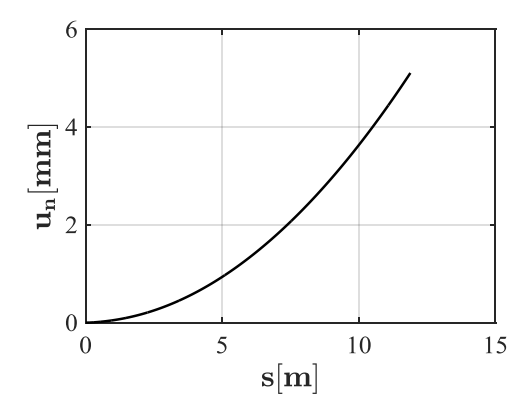

b) Displacement in n-direction

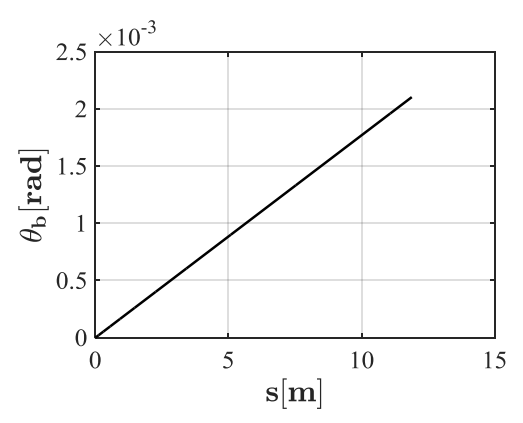

c) Rotation about b-axis

Figure 12: Logarithmic spiral - displacements and rotation along arc-length in local coordinate system.

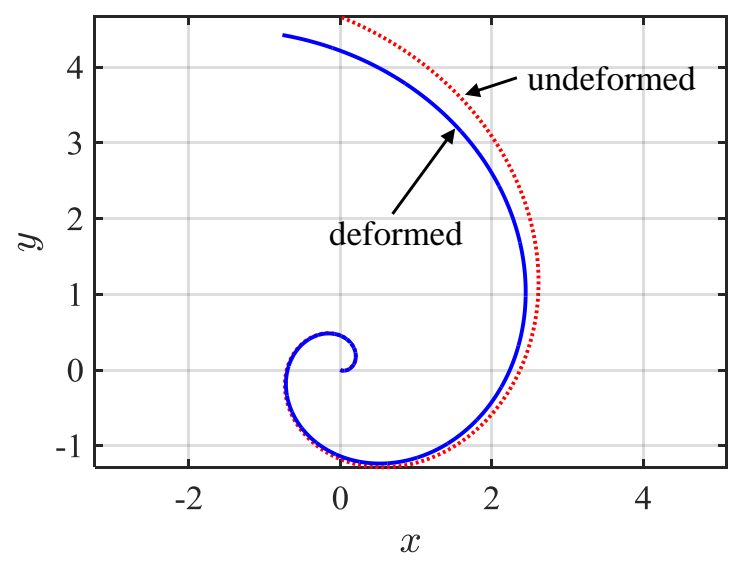

Figure 13: Logarithmic spiral - reference and deformed shape.

\subsection{Spiral staircase}


The third example is a 3-D helical spiral staircase parameterized by:

$$
\boldsymbol{\alpha}(s)=\left\{a \cos \left(\frac{S}{c}\right), a \sin \left(\frac{S}{c}\right), b \frac{s}{c}\right\}
$$

with $a=2, b=\frac{3}{2 \pi}$ and $c=\sqrt{a^{2}+b^{2}}=2.06$. The geometry and loading are shown in Figure 14, where the parameters used result in a total height $H$ of $3 \mathrm{~m}$ and radius of $2 \mathrm{~m}$. The distributed load $q$ is $3 \mathrm{kN} / \mathrm{m}$ in the $(-) \mathrm{z}$-direction and the bottom end is fully fixed. The hollow circular crosssection has an outer radius $r=15 \mathrm{~cm}$, thickness $t=4 \mathrm{~cm}$ and $\varepsilon^{2}=5.18 \times 10^{-5}$. Because the curve is a helix, both the curvature $(\kappa=0.473)$ and torsion $(\tau=0.113)$ are constant along the arclength. A $3^{\text {rd }}$ degree NURBS basis with 264 elements are used for the analysis, and the nonzero internal forces and moments in the local coordinate system along the arc-length are shown in Figure 15. Figure 16 shows the displacements and rotations in the local coordinate system along the arc-length and Figure 17 gives the reference (red dotted line) and deformed (blue solid line) shapes with a magnification factor of 10 used for the deformed shape.

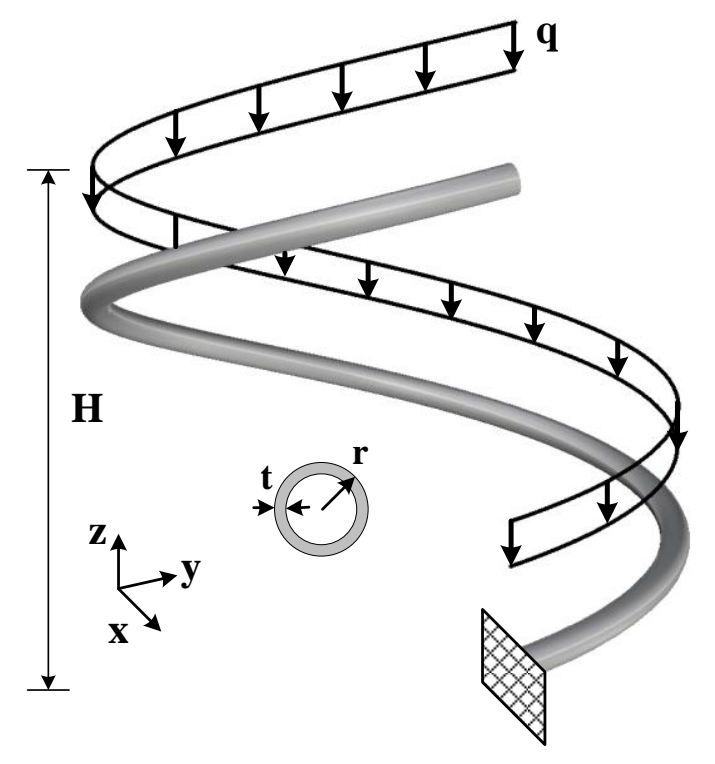

Figure 14: Spiral staircase - geometry and loading.

To further validate the implementation of 3-D NURBS curved beam, a convergence study for the vertical displacement in global system is carried out for various order of basis as shown in Figure 
18. The numerically exact solution shown in Figure 18 is obtained by solving the differential equations directly in the global coordinates [12], using the solver for boundary value problems for ordinary differential equations $b v p 4 c(\cdot)$ in Matlab ${ }^{\circledR}$. Figure 18 shows that as the mesh is refined, convergence can be obtained towards the numerically exact solution and the higher order basis gives better convergence, as expected. This exercise further validates the proposed 3-D curved NURBS based beam formulation and implementation.

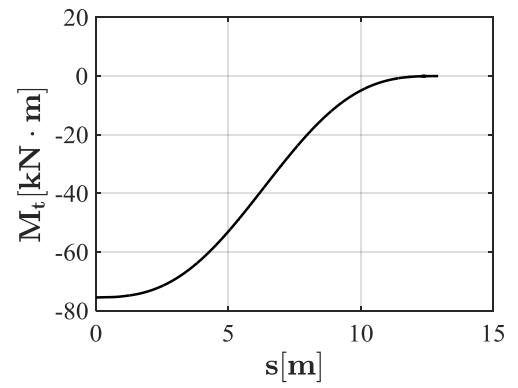

a) Moment about t-axis

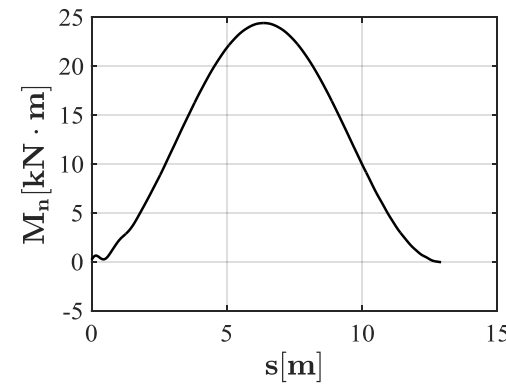

b) Moment about n-axis

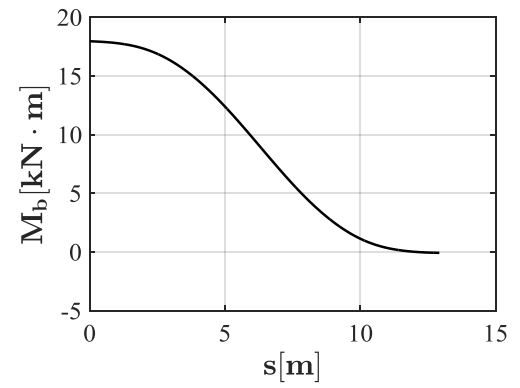

c) Moment about b-axis

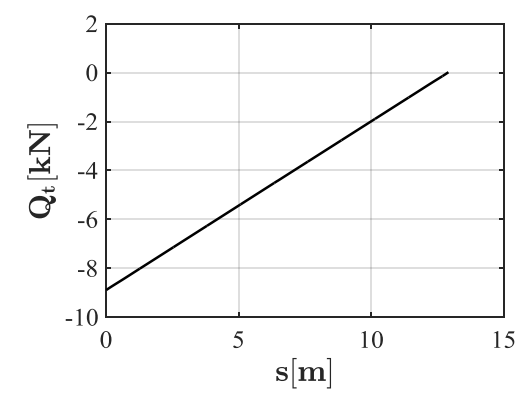

d) Axial force in t-direction

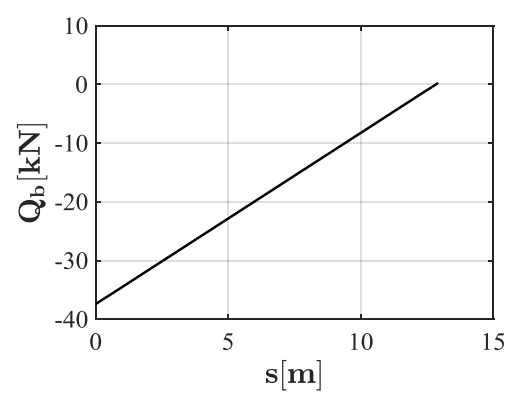

e) Shear force in b-direction

Figure 15: Spiral staircase - internal moments and forces along arc-length in local coordinate system.

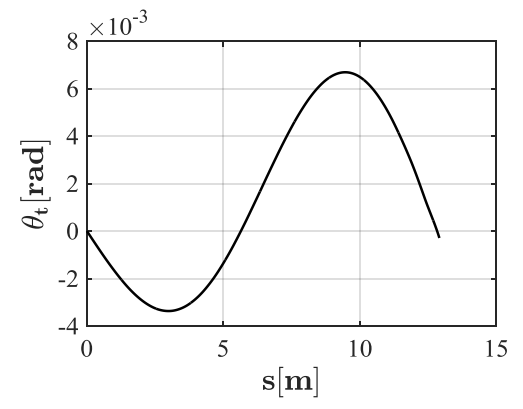

a) Rotation about t-axis

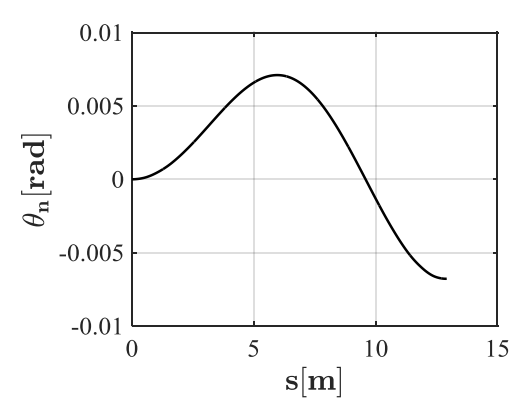

b) Rotation about n-axis

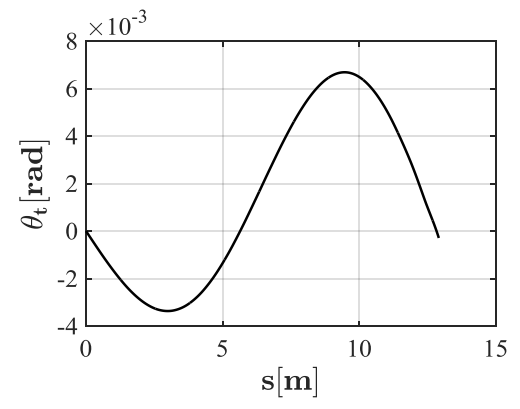

c) Rotation about b-axis 


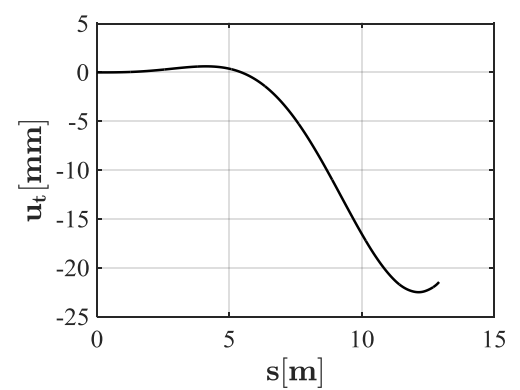

d) Displacement in t-direction

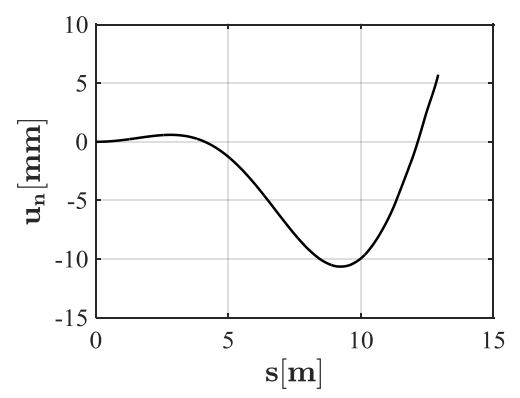

e) Displacement in n-direction

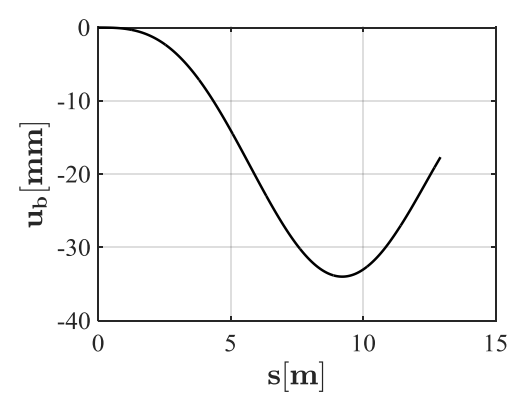

f) Displacement in b-direction

Figure 16: Spiral staircase - rotations and displacements along arc-length in local coordinate system.

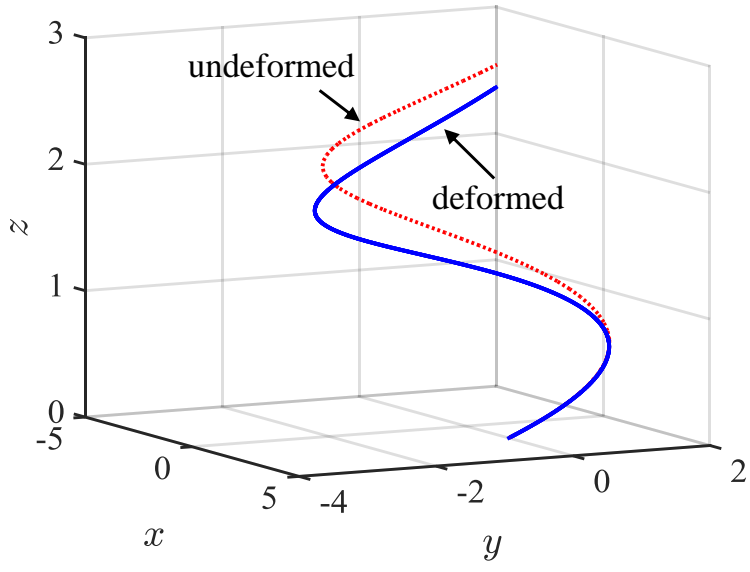

Figure 17: Spiral staircase - reference and deformed shape.

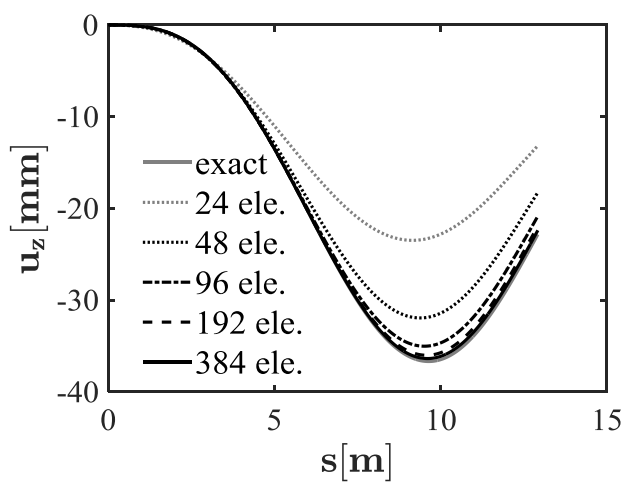

a) Cubic NURBS basis

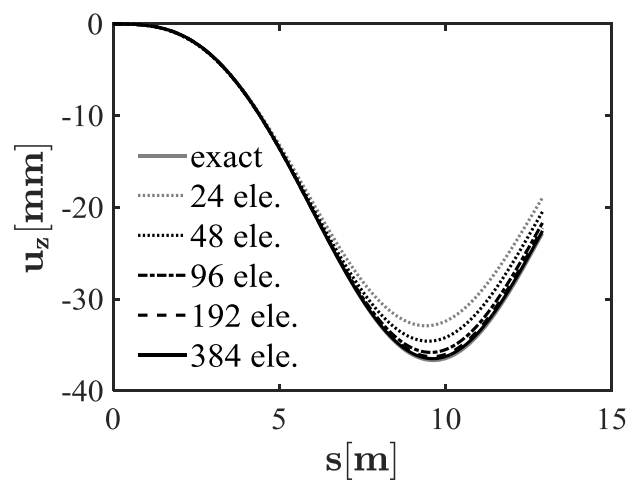

b) Fourth degree NURBS basis 


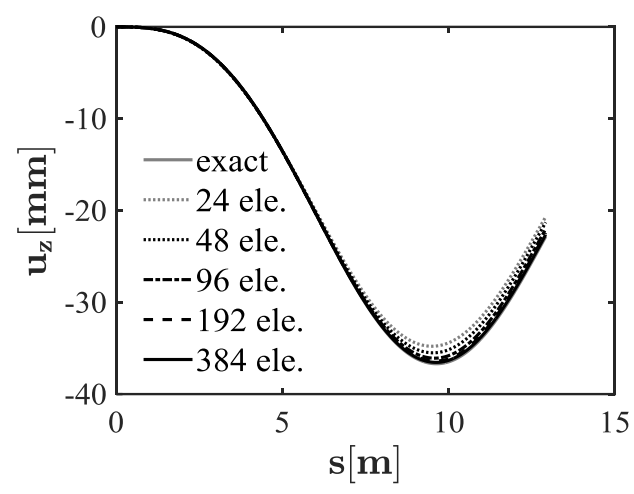

c) Fifth degree NURBS basis

Figure 18: Convergence study for 3-D spiral staircase.

\subsection{Out of plane arch}

This example consists of a circular arch that is pulled out of plane. The geometry is shown in Figure 19 where $L=10 \mathrm{~m}$, the height $H=8.75 \mathrm{~m}$ and the distance out of plane is $D=6.38 \mathrm{~m}$. The control points and weights of the coarsest mesh to construct the curve are shown in Table 1 and the corresponding knot vector is $\Xi=\{0,0,0,0,0,1,1,1,1,1\}$. The structure is subject to a distributed load of $5 \mathrm{kN} / \mathrm{m}$ in the (-) $z$-direction shown in Figure 19 and both ends are fully fixed. The cross-section used is again a hollow circle, with an outer radius of $20 \mathrm{~cm}$, thickness of $10 \mathrm{~cm}$ and $\varepsilon^{2}=1.13 \times 10^{-5}$. Unlike the previous example, both curvature and torsion change along the arc-length in this example. The curvature and torsion are plotted as a function of arc-length in Figure 20. Analysis is carried out with a $4^{\text {th }}$ degree NURBS basis and 51 Bézier elements. Internal forces and moments in the local coordinate system along the arc-length are shown in Figure 21. The rotations and displacements in the local coordinate system along arc-length are shown in Figure 22, and the reference (red line) and deformed (blue line) shapes with a magnification factor of 50 are given in Figure 23. 


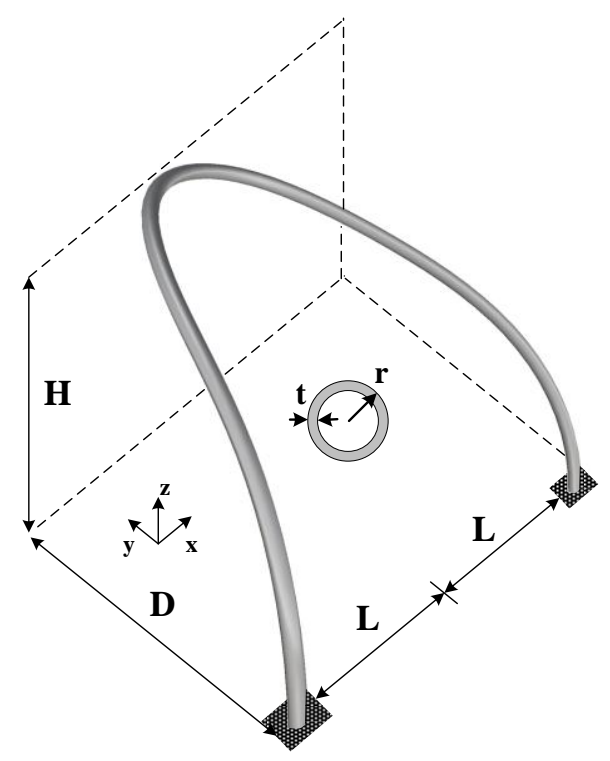

Figure 19: Out of plane arch geometry.

Table 1: Control points and weights for the out of plane arch.

\begin{tabular}{ccc}
\hline \hline$q$ & $\boldsymbol{C}_{q}$ & $w_{q}$ \\
\hline \hline 1 & $(0,10,0)$ & 1.0 \\
2 & $(0,10,10)$ & 1.0 \\
3 & $(17,0,10)$ & 1.0 \\
4 & $(0,-10,10)$ & 1.0 \\
5 & $(0,-10,0)$ & 1.0 \\
\hline
\end{tabular}

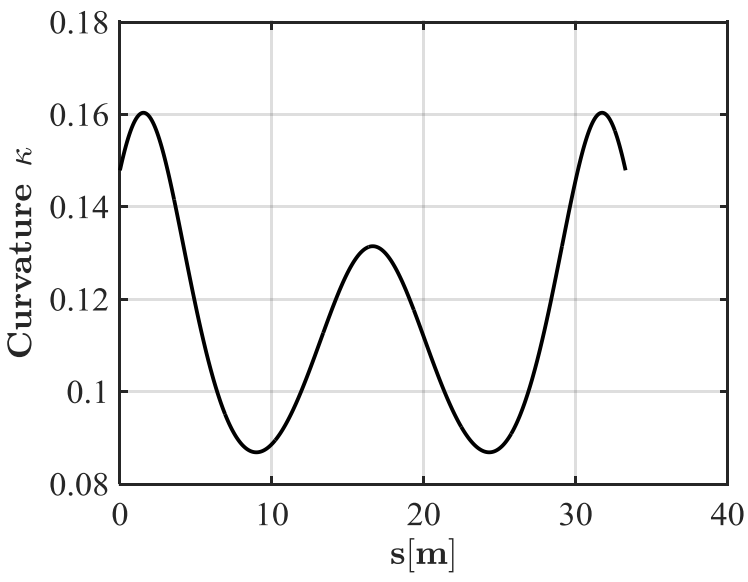

a) Curvature

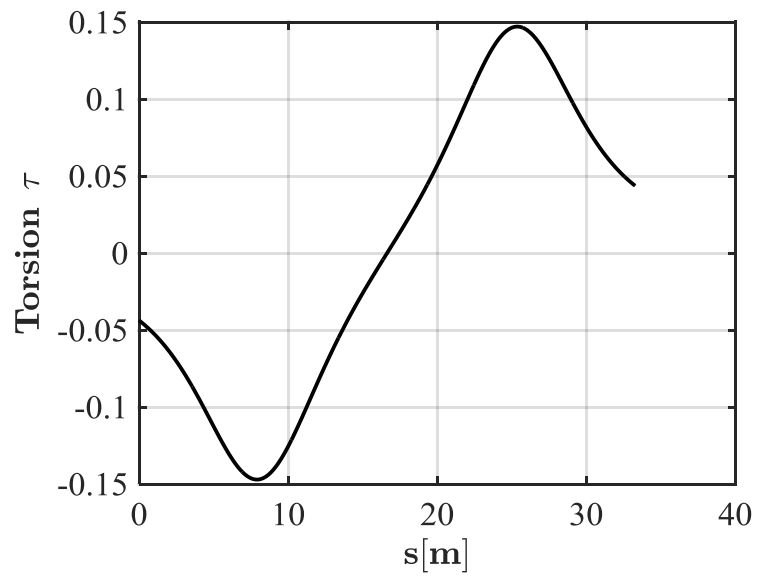

b) Torsion

Figure 20: Curvature and torsion as a function of arc-length for the out of plane arch. 


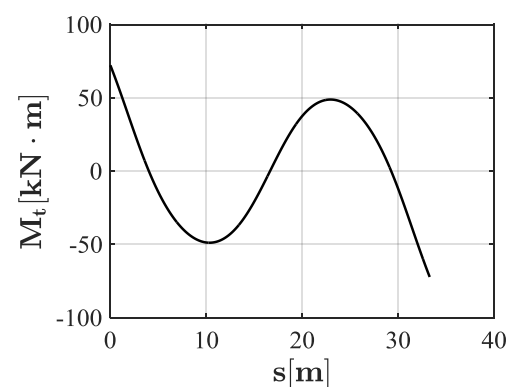

a) Moment about t-axis

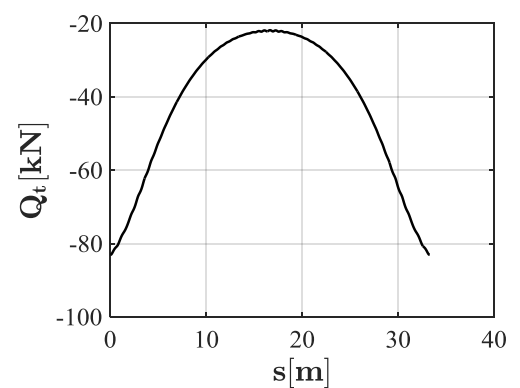

d) Axial force in t-direction

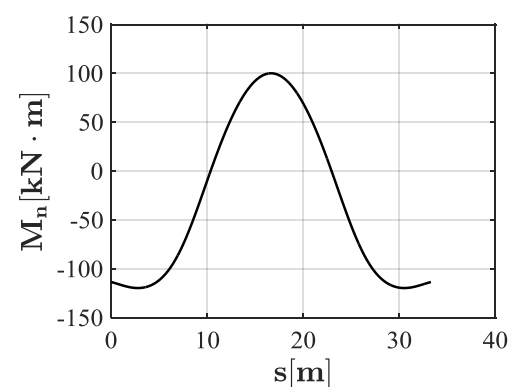

b) Moment about n-axis

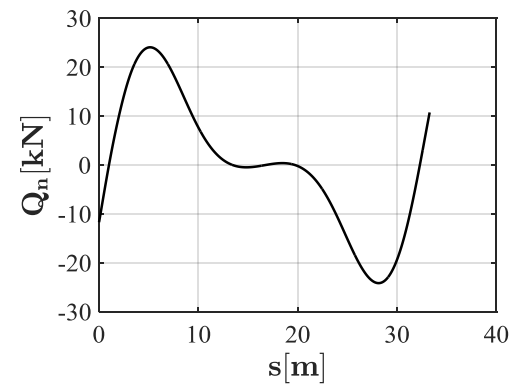

e) Shear force in n-direction

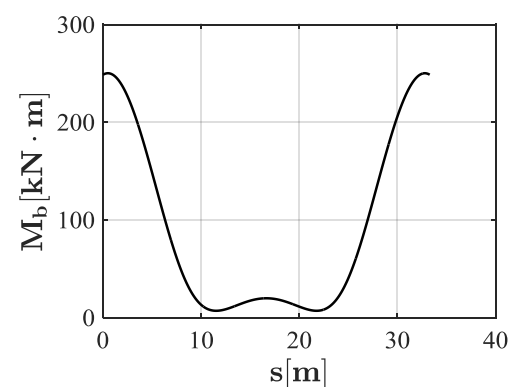

c) Moment about b-axis

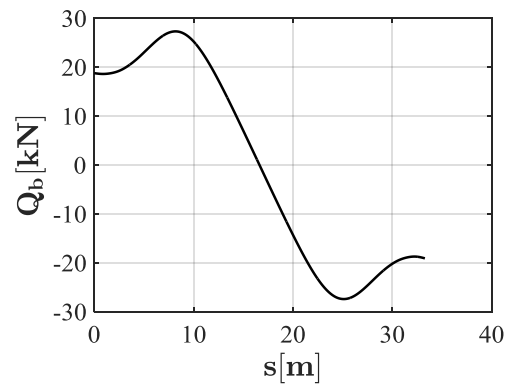

f) Shear force in b-direction

Figure 21: Out of plane arch - internal moments and forces along arc-length in local coordinate system.

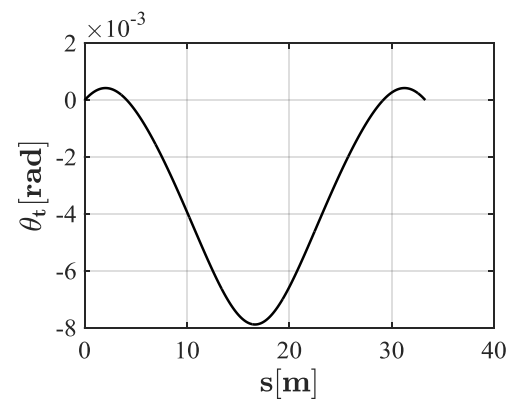

a) Rotation about t-axis

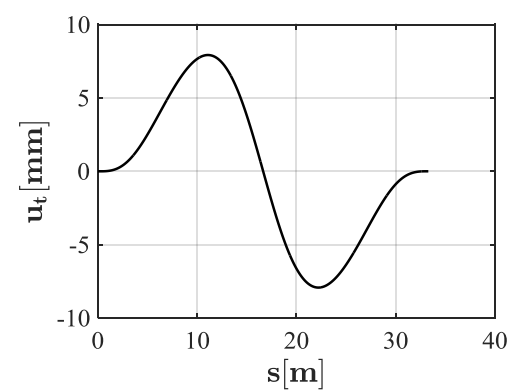

d) Displacement in t-direction

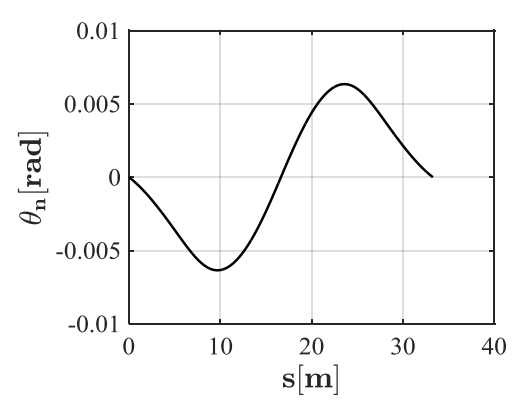

b) Rotation about n-axis

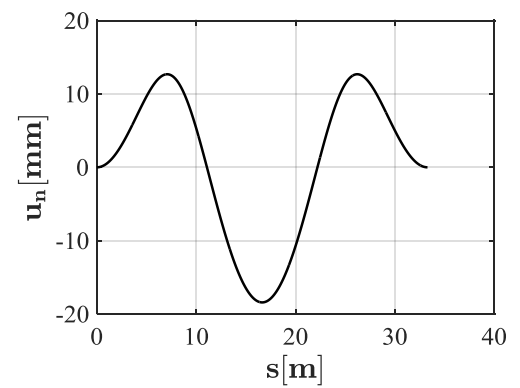

e)Displacement in n-direction

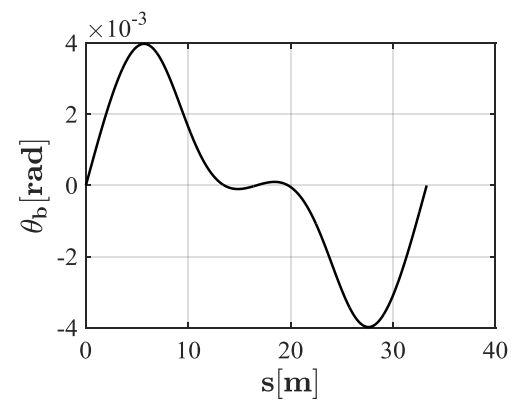

c) Rotation about b-axis

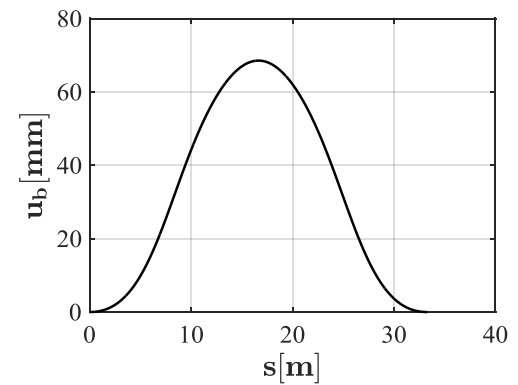

f) Displacement in b-direction Figure 22: Out of plane arch - rotations and displacements along arc-length in local coordinate system. 


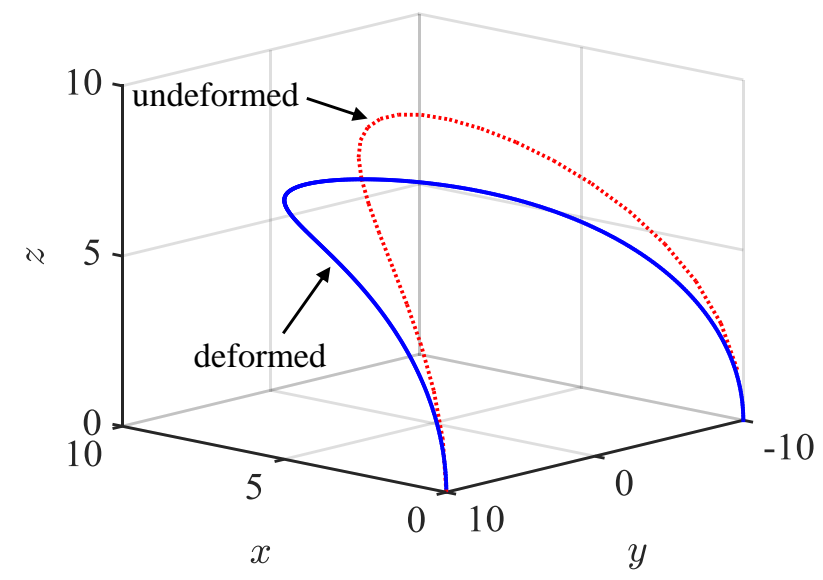

Figure 23: Out of plane arch - reference and deformed shape.

\subsection{S-curved beam}

In the last example, an S-curved beam shown in Figure 24 is studied. The radius of the quarter circle is $5 \mathrm{~m}$ making the horizontal and vertical span $H=L=10 \mathrm{~m}$. The cross-section considered is again a hollow section with an outer radius of $15 \mathrm{~cm}$ and thickness of $4 \mathrm{~cm}$ and $\varepsilon^{2}=3.51 \times$ $10^{-5}$. A constant external horizontal distributed load $q=0.5 \mathrm{kN} / \mathrm{m}$ is acting along the $\mathrm{S}$-curved cantilever beam.

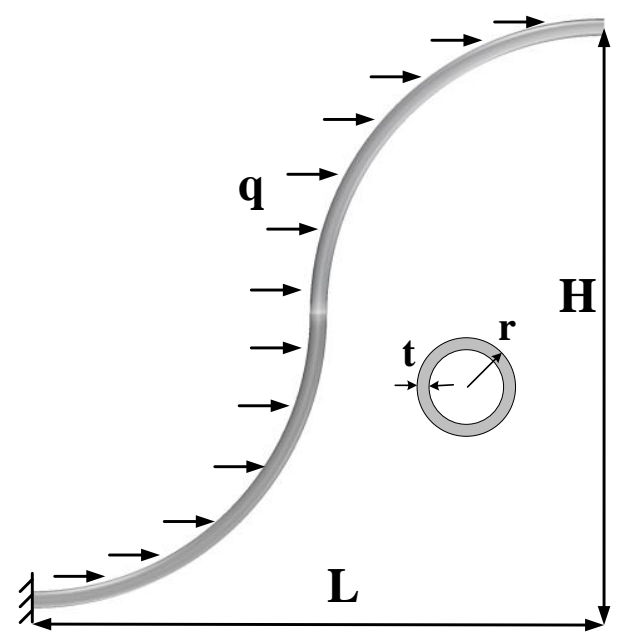

Figure 24: S curve - geometry and loading. 


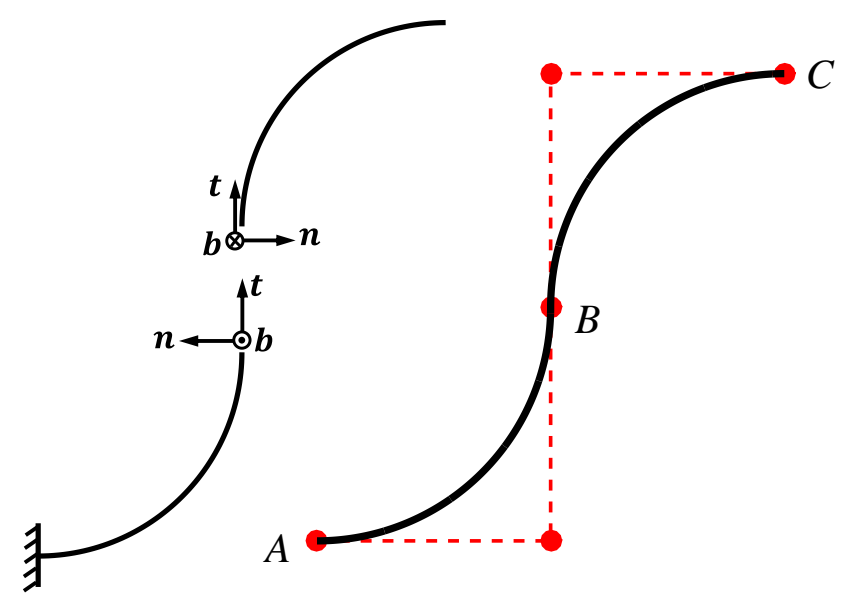

Figure 25: Non smoothness of the Frenet-Serret basis at Point B on the beam.

With this geometry, the Frenet-Serret frame cannot be uniquely determined at all the points on the curve. More precisely, the normal direction $\boldsymbol{n}$ flips at point $B$, where the curvature switches the direction (Figure 25). To handle this case, the S-curved beam is divided into two patches with each patch representing a quarter circle and thus the point $B$ becomes interpolatory in both patches. Due to the non-uniqueness of local Frenet-Serret basis frame at $B$, the unknown variables at this control point are projected from the local coordinate system in each patch to the global coordinate system during the finite element assembly process. Thus, at the control point $B$, the unknown variables are defined in the global coordinate system. Analysis is carried out with a $4^{\text {th }}$ degree NURBS basis and 26 Bezier elements. The internal forces and moment in both local and global coordinate systems are shown in Figure 26. It can be seen that shear force $Q_{n}$ and moment $M_{b}$ are discontinuous at $B$ due to the discontinuity of the Frenet-Serret basis, whereas in the global system both $Q_{x}$ and $M_{z}$ are continuous. Figure 27 gives the displacements and rotations in both local and global systems in which similar discontinuities can be observed for the local variables $\left(u_{n}\right.$ and $\left.\theta_{b}\right)$, while the description in global coordinate system is continuous. The deformed (blue line) shape with a magnification factor of 50 together with the reference geometry are provided in Figure 28. 


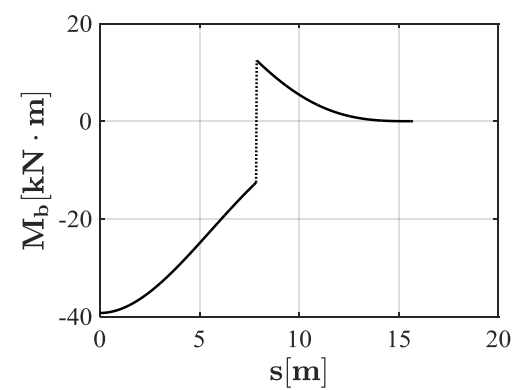

a) Moment about b-axis

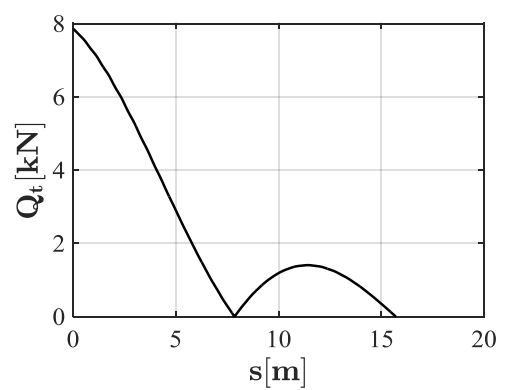

b) Axial force in t-direction

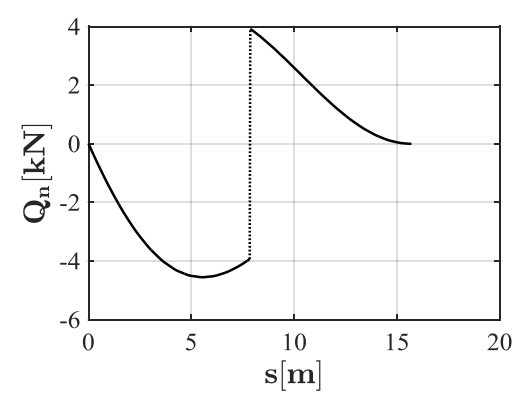

c) Shear force in n-direction

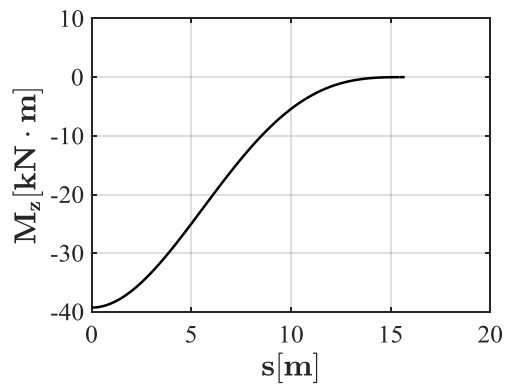

d) Moment about z-axis

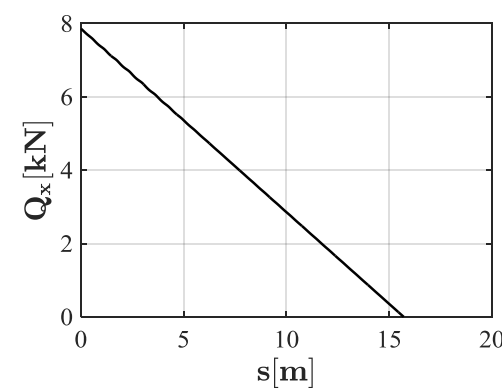

e) Internal force in $\mathrm{x}$-direction

Figure 26: $\mathrm{S}$ curve - internal moments and forces along arc-length in local and global coordinate system.

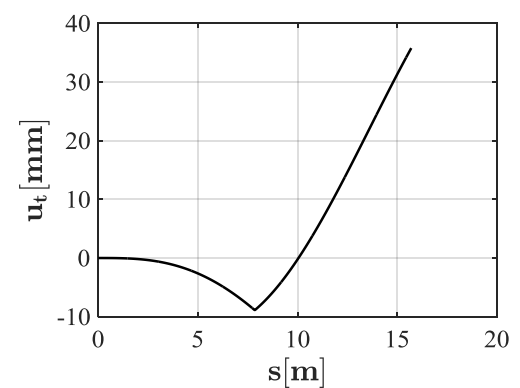

a) Displacement in t-direction

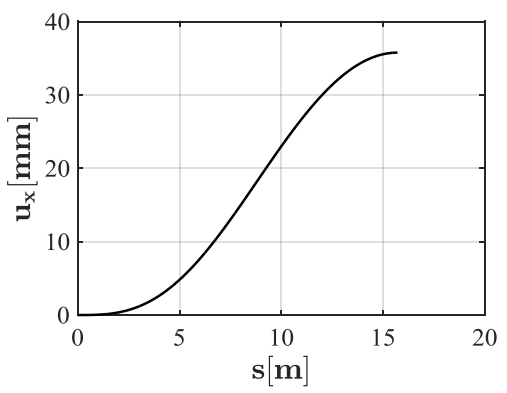

a) Displacement in $\mathrm{x}$-direction

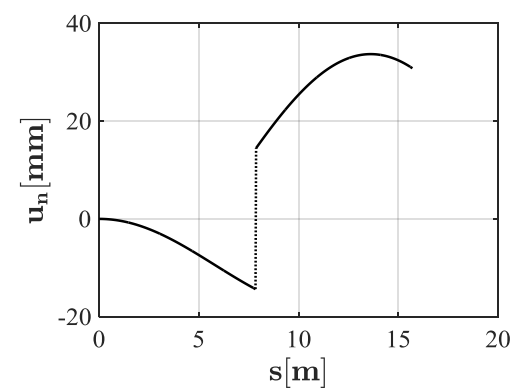

b)Displacement in n-direction

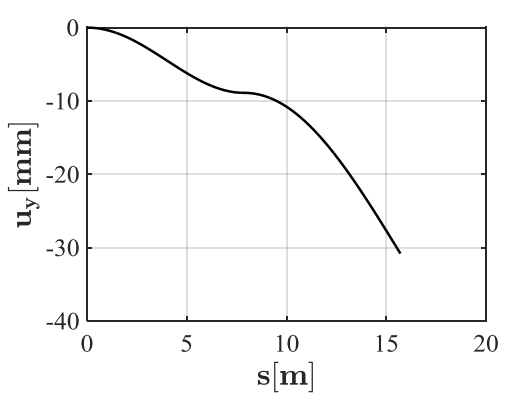

b) Displacement in y-direction

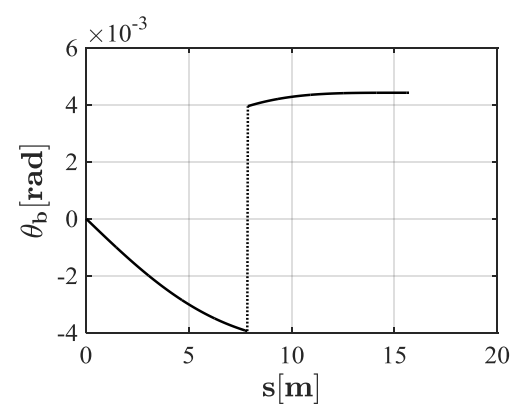

c) Rotation about b-axis

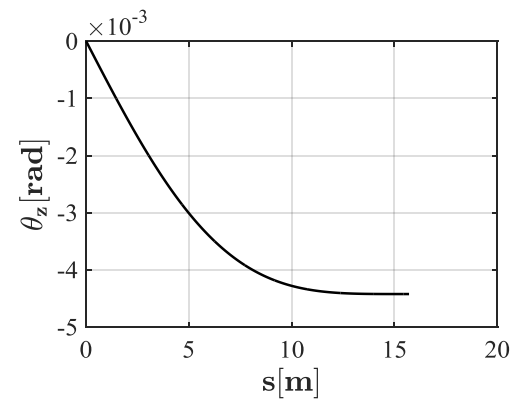

c) Rotation about z-axis

Figure 27: S curve - rotations and displacements along arc-length in local and global coordinate system. 


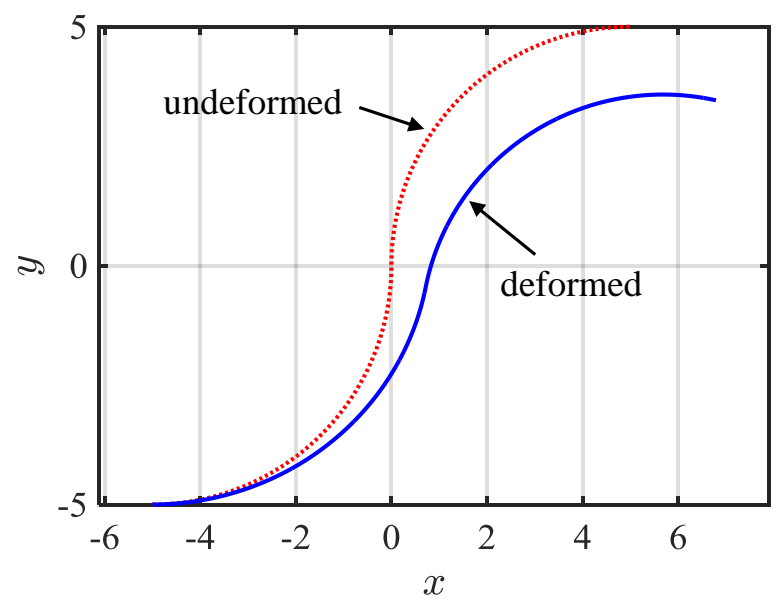

Figure 28: $\mathrm{S}$ curve - reference and deformed shape.

\section{Conclusions}

In this study, isogeometric analysis is used to formulate finite elements for arbitrary spatially curved beams. The isogeometric approach allows for a seamless transition between CAD and structural analysis since the geometrical representations used in the CAD model are taken as the basis for interpolation of geometry and unknown fields in the isogeometric analysis. The shear and membrane locking issues in curved beam elements are addressed by increasing the order of interpolations used in the analysis. In future work, the issue of locking will be more thoroughly investigated for lower order NURBS basis. A variety of examples of curved beams with varying complexity are analyzed to show the efficacy of the proposed approach. These examples demonstrate the ability of the proposed approach to deal with complex geometrical forms, and can be used by architects and engineers to design and discover novel structural forms. It is important to note that the isogeometric method can be also used for shape optimization of curved structures as the shape sensitivity analyses can be directly carried out on the CAD geometries. Moreover, only the small deformation theory with linear elastic material is considered in this study; however, the presented IGA framework can be extended to nonlinear beam theories. Further work is in progress to address these important topics. 


\section{Acknowledgements}

The presented work is supported in part by the US National Science Foundation through grant CMS-1055314. Any opinions, findings, conclusions, and recommendations expressed in this paper are those of the authors and do not necessarily reflect the views of the sponsors. 


\section{References}

[1] Dawe DJ. Matrix and Finite Element Displacement Analysis of Structures: Clarendon Press, Oxford; 1984.

[2] Piegl L, Tiller W. The NURBS book: Springer Science \& Business Media; 2012.

[3] Hughes TJR, Cottrell JA, Bazilevs Y. Isogeometric analysis: CAD, finite elements, NURBS, exact geometry and mesh refinement. Computer Methods in Applied Mechanics and Engineering. 2005;194:4135-95.

[4] Cottrell JA, Hughes TJ, Bazilevs Y. Isogeometric analysis: toward integration of CAD and FEA: John Wiley \& Sons; 2009.

[5] Verhoosel CV, Scott MA, Hughes TJ, De Borst R. An isogeometric analysis approach to gradient damage models. International Journal for Numerical Methods in Engineering. 2011;86:115-34.

[6] Zhang G, Khandelwal K. Modeling of nonlocal damage-plasticity in beams using isogeometric analysis. Computers \& Structures. 2016;165:76-95.

[7] Wall WA, Frenzel MA, Cyron C. Isogeometric structural shape optimization. Computer Methods in Applied Mechanics and Engineering. 2008;197:2976-88.

[8] Cottrell JA, Reali A, Bazilevs Y, Hughes TJR. Isogeometric analysis of structural vibrations. Computer Methods in Applied Mechanics and Engineering. 2006;195:5257-96.

[9] Zhang G, Li L, Khandelwal K. Nonlinear forced vibration of timoshenko micro-beams using isogeometric analysis. Computers \& Structures (Submitted). 2015.

[10] Palaninathan R, Chandrasekharan PS. Curved beam element stiffness matrix formulation. Computers \& Structures. 1985;21:663-9.

[11] Marquis JP, Wang TM. Stiffness matrix of parabolic beam element. Computers \& Structures. 1989;31:863-70.

[12] Gimena L, Gimena FN, Gonzaga P. Structural analysis of a curved beam element defined in global coordinates. Engineering Structures. 2008;30:3355-64.

[13] Arunakirinathar K, Reddy BD. Mixed finite element methods for elastic rods of arbitrary geometry. Numerische Mathematik. 1993;64:13-43.

[14] Loula AFD, Franca LP, Hughes TJR, Miranda I. Stability, convergence and accuracy of a new finite element method for the circular arch problem. Computer Methods in Applied Mechanics and Engineering. 1987;63:281-303.

[15] Benedetti A, Tralli A. A new hybrid F.E. model for arbitrarily curved beam-I. Linear analysis. Computers \& Structures. 1989;33:1437-49. 
[16] Kim JG, Kim YY. A new higher-order hybrid-mixed curved beam element. International Journal for Numerical Methods in Engineering. 1998;43:925-40.

[17] Adam C, Bouabdallah S, Zarroug M, Maitournam H. Improved numerical integration for locking treatment in isogeometric structural elements, Part I: Beams. Computer Methods in Applied Mechanics and Engineering. 2014;279:1-28.

[18] Bouclier R, Elguedj T, Combescure A. Locking free isogeometric formulations of curved thick beams. Computer Methods in Applied Mechanics and Engineering. 2012;245-246:144-62.

[19] Abbena E, Salamon S, Gray A. Modern differential geometry of curves and surfaces with Mathematica: CRC press; 2006.

[20] Tabarrok B, Farshad M, Yi H. Finite element formulation of spatially curved and twisted rods. Computer Methods in Applied Mechanics and Engineering. 1988;70:275-99.

[21] Cox MG. The Numerical Evaluation of B-Splines. IMA Journal of Applied Mathematics. 1972;10:134-49.

[22] de Boor C. On calculating with B-splines. Journal of Approximation Theory. 1972;6:50-62.

[23] Borden MJ, Scott MA, Evans JA, Hughes TJ. Isogeometric finite element data structures based on Bézier extraction of NURBS. International Journal for Numerical Methods in Engineering. 2011;87:15-47.

[24] Rhinoseros (https://www.rhino3d.com/); Robert McNeel \& Associates. 2015.

[25] Prathap G. The Finite Element Method in Structural Mechanics: Springer Netherlands; 1993.

[26] Echter R, Bischoff M. Numerical efficiency, locking and unlocking of NURBS finite elements. Computer Methods in Applied Mechanics and Engineering. 2010;199:374-82.

[27] Elguedj T, Bazilevs Y, Calo VM, Hughes TJR. B-bar and F-bar projection methods for nearly incompressible linear and non-linear elasticity and plasticity using higher-order NURBS elements. Computer Methods in Applied Mechanics and Engineering. 2008;197:2732-62. 